\title{
Beables/Observables in Classical and Quantum Gravity
}

\author{
Edward ANDERSON
}

DAMTP, Centre for Mathematical Sciences, Wilberforce Road, Cambridge CB3 OWA, UK

E-mail: ea212@cam.ac.uk

Received December 20, 2013, in final form August 18, 2014; Published online August 29, 2014

http://dx.doi.org/10.3842/SIGMA.2014.092

\begin{abstract}
Observables 'are observed' whereas beables just 'are'. This gives beables more scope in the cosmological and quantum domains. Both observables and beables are entities that form 'brackets' with 'the constraints' that are 'equal to' zero. We explain how depending on circumstances, these could be, e.g., Poisson, Dirac, commutator, histories, Schouten-Nijenhuis, double or Nambu brackets, first-class, gauge, linear or effective constraints, and strong, weak or weak-effective equalities. The Dirac-Bergmann distinction in notions of gauge leads to further notions of observables or beables, and is tied to a number of diffeomorphism-specific subtleties. Thus we cover a wide range of notions of observables or beables that occur in classical and quantum gravitational theories: Dirac, Kuchař, effective, Bergmann, histories, multisymplectic, master, Nambu and bi-. Indeed this review covers a representatively wide range of such theories: general relativity, loop quantum gravity, histories theory, supergravity and M-theory.
\end{abstract}

Key words: observables; classical and quantum gravity; problem of time; constrained dynamics

2010 Mathematics Subject Classification: 83C05; 83C45; 83D05; 70H45; 81S05

\section{Introduction}

This review covers a topic - observables and beables - which spans classical dynamics and quantum mechanics, with the canonical perspective of each of quantum cosmology and quantum gravity particularly in mind.

Observables/beables [38, 39, 55, 56, 57, 58, 71, 88, 93, 106, 107, 131, 137, 155, 152] are often considered to be objects whose 'brackets' with 'the constraints' are 'equal to' zero:

$$
\left|\left[\mathcal{C}_{\mathrm{C}}, B_{\mathrm{B}}\right]\right|^{\prime}={ }^{\prime} 0 .
$$

Here $\mathcal{C}_{\mathrm{C}}$ denotes the constraints and $B_{\mathrm{B}}$ denotes the beables; $\mathrm{C}$ and $\mathrm{B}$ index for now general sets of each of these. $|[]$,$| is usually a Lie bracket such as a Poisson bracket in classical dynamics$ or a quantum commutator. As a Lie bracket, it obeys the Jacobi identity

$$
|[X,|[Y, Z]|]|+\text { cycles }=0 .
$$

However, there are a number of different possibilities for which brackets, which constraints and even which notion of equality can be involved. Thus we will first need to discuss each of these more primary entities (Sections 1.1-1.7) with additionally some types of constraint having ties to notions of gauge. Additionally, there are notions of gauge not tied to constraints which furnish a further conception of observables/beables along the lines of Bergmann [38, 39]. After this, we can return to considering the more composite notions that are observables and beables, in Sections 1.8-1.10. 
We do first consider the distinction between observables and beables. This began with Bell [36], and is the difference between entities being observed and entities simply being. The circumstances under which observables occur are then a subset of those in which beables do, in the sense that 'being observed' is a subset of 'being'. Moreover, from a beables perspective, defining what 'observing' is is unnecessary, so conceptualizing in terms of beables is a freeing from having to define this.

Two contexts in which beables are relevant are 1) whole-universe or closed-system modelling, and 2) at the quantum level. Bell pointed out that 1) is already an issue at the classical level [34]. This is due to observers living within such a universe rather than affording a 'God's eye' view from outside. (Also observers did not exist in the early universe.) On the other hand, 2) has more widespread relevance due to the connection between the notion of observation and the quantum measurement problem [142, 165]; Bell furthermore extended the notion of beables to QFT in [35].

Along lines e.g. recently argued for by Kent [102], beables are furthermore an appropriate concept for a number of types of realist interpretation of QM. (This is as opposed to e.g. instrumentalist interpretation; see e.g. [94] for an introduction to different types of interpretation of QM.) The Bohmian approach is one branch of realist interpretations in which the name and concept of 'beables' is widely used [31, 35, 45, 51, 72, 83, 116, 139, 148, 145, 162]. Moreover, histories approaches (see [70, 84, 97], or Appendix A for a summary) can also be thought of in terms of beables. The beables concept additionally comes hand-in-hand with QM wavefunction collapse due to decoherence [100] by natural phenomena as opposed to by observation. I.e. in the Universe, processes such as dust grains being decohered by CMB photons [99] are more typical than processes specifically involving observers making quantum measurements. Finally, beables are also appropriate in the contextual realist interpretation of QM by Isham and Doering [61] ${ }^{1}$. Among these realist approaches, histories and decoherence play further role in this review.

As a third context combining the previous two, quantum cosmology is substantially distinct from QM. The measurement problem is further aggravated in this setting, for which the usual Copenhagen interpretation of QM can no longer apply. Quantum cosmology has its own distinct conceptualization of histories and decoherence [77, 84, 100]. Yet the concept of beables continues to be appropriate in quantum cosmology.

Henceforth I use 'beables' unless the situation specifically requires use of the word 'observables'.

\subsection{Outline of constrained dynamics and various kinds of constraints}

Denote the generalized configurations of a physical system by $Q^{\mathrm{A}}{ }^{2}{ }^{2}$ E.g. particle positions in mechanics, field values in field theory, or spatial 3-metrics $h_{i j}$ on a fixed topological manifold $\Sigma$ in the geometrodynamical formulation of general relativity (GR): Wheeler's [168] formulation of GR as evolving spatial 3-geometries. The space of possible values that the $Q^{\mathrm{A}}$ can take is the configuration space $\mathfrak{q}[110]$, e.g. $\mathbb{R}^{N d}$ for $N$ particles in dimension $d$, or the space $\operatorname{Riem}(\Sigma)$ of $h_{i j}$ 's for geometrodynamics. A given (for now finite) classical physical system's equations of motion can be taken to follow from the Lagrangian $\mathrm{L}\left(Q^{\mathrm{A}}, \dot{Q}^{\mathrm{A}}\right) \cdot{ }^{3}$ The $Q^{\mathrm{A}}$ then have conjugate

\footnotetext{
${ }^{1}$ This approach is based on multi-valued context-dependent truth valuations via use of Topos Theory to reinterpret the foundations of QM. As such this approach is given here as motivation for realist approaches, but further details of it lie beyond the scope of this review.

${ }^{2}$ In this review, sans-serif capital letters are used as generalized indices, lower-case Latin letters are used for spatial indices, and lower-case Greek letters for spacetime ones. Primed and unprimed indices index the same objects throughout this review. Following [93, 106], I use ( ) for functions, [ ] for functionals, and ( ; ] for mixed function-functionals. This leaves \{\} without commas for actual brackets. I then use bold font to clearly distinguish Poisson brackets $\{$,$\} and other brackets playing analogous roles in defining notions of beables.$

${ }^{3}$ Here ${ }^{\prime}$ is $\partial / \partial t$ for $t$ a notion of time for one's theory, which includes in some cases $\partial / \partial \lambda$ for $\lambda$ a meaningless label time. This formula and the rest in this section are for finite models such as mechanics or minisuperspace (homogeneous GR), but have well-known extensions to field theories (including GR and alternative theories of gravity).
} 
momenta

$$
P_{\mathrm{A}}:=\partial \mathrm{L} / \partial \dot{Q}^{\mathrm{A}}
$$

One can additionally pass (via a so-called Legendre transformation) from $Q^{\mathrm{A}}$ and $\dot{Q}^{\mathrm{A}}$ variables and a Lagrangian function of these, $\mathrm{L}\left(Q^{\mathrm{A}}, \dot{Q}^{\mathrm{A}}\right)$, to $Q^{\mathrm{A}}$ and $P_{\mathrm{A}}$ variables and a Hamiltonian function of these, $\mathrm{H}\left(Q^{\mathrm{A}}, P_{\mathrm{A}}\right)$. Phase space is the space of both the $Q^{\mathrm{A}}$ and the $P_{\mathrm{A}}$ as equipped with the Poisson brackets

$$
\{F, G\}:=\frac{\partial F}{\partial Q^{\mathrm{A}}} \frac{\partial G}{\partial P_{\mathrm{A}}}-\frac{\partial F}{\partial P_{\mathrm{A}}} \frac{\partial G}{\partial Q^{\mathrm{A}}} .
$$

From a more geometrical perspective, Poisson brackets are well-known to be recastable in terms of a symplectic form [17]. These notions readily extend to field theories by upgrading to suitable functionals and including suitable integrals over one's notion of space.

Moreover, passage from a Lagrangian perspective to a Hamiltonian one can be nontrivial. Furthermore, it is the Hamiltonian perspective which possesses a systematic treatment of constraints, due to Dirac [56, 88]. The Hamiltonian perspective additionally offers a more direct link to quantum theory. The above nontriviality is due to the array

$$
\partial^{2} \mathrm{~L} / \partial \dot{Q}^{\mathrm{A}} \partial \dot{Q}^{\mathrm{A}^{\prime}} \quad\left(=\partial P_{\mathrm{A}^{\prime}} / \partial \dot{Q}^{\mathrm{A}}\right)
$$

- associated with the Legendre transformation - in general being non-invertible, by which the momenta cannot be independent functions of the velocities. Thus there are relations $\mathcal{C}_{\mathrm{C}}\left(Q^{\mathrm{A}}, P_{\mathrm{A}}\right)=0$ between the momenta; these are standardly termed constraints. (In this review, constraints are highlighted by exclusive use of the calligraphic font.) Moreover, the above array also features in the reformulation of the Euler-Lagrange equations as

$$
\ddot{Q}^{\mathrm{A}^{\prime}} \partial^{2} \mathrm{~L} / \partial \dot{Q}^{\mathrm{A}} \partial \dot{Q}^{\mathrm{A}^{\prime}}=\partial \mathrm{L} / \partial Q^{\mathrm{A}}-\dot{Q}^{\mathrm{A}^{\prime}} \partial^{2} \mathrm{~L} / \partial Q^{\mathrm{A}} \partial \dot{Q}^{\mathrm{A}^{\prime}} .
$$

Due to this, the noninvertibility has additional significance as accelerations not being uniquely determined by $Q^{A}, \dot{Q}^{\mathrm{A}}$. $^{4}$

Constraints are usefully classified in a number of ways, including the following due to Bergmann and Dirac [56, 88].

Primary constraints arise purely from the form of the Lagrangian; these are the relations between the momenta by which the above-mentioned Legendre transformation maps onto only a submanifold of the full phase space.

Secondary constraints, on the other hand, arise via use of the equations of motion. One intuitively valuable case of this concerns constraints arising from the propagation of existing constraints using the equations of motion.

Weak equality is equality up to additive functionals of the constraints; this holds on the constraint surface (defined as the surface within phase space where the totality of the constraints vanishes).

First-class constraints are then those whose classical brackets with all the other constraints vanish weakly; these are indexed by $F$. This can also be described in terms of no new entities constraints or further kinds mentioned below - arising from the bracket operation acting on $\mathcal{C}_{\mathrm{F}}$ and a general $\mathcal{C}_{\mathrm{C}}$. Geometrically, these are characterized as the brackets that vanish on the version of constraint surface upon which all first-class constraints vanish. Ab initio, the classical brackets involved are Poisson brackets.

Second-class constraints are then simply defined by exclusion as those constraints that fail to be first-class.

\footnotetext{
${ }^{4}$ For simplicity, this review's range of physical theories restricts itself to no higher than second-order theories.
} 
Moreover, one can always in principle handle second-class constraints by passing from Poisson to Dirac brackets,

$$
\{F, G\}^{*}:=\{F, G\}-\left\{F, \mathcal{C}_{\mathrm{S}}\right\}\left\{\mathcal{C}_{\mathrm{S}}, \mathcal{C}_{\mathrm{S}^{\prime}}\right\}^{-1}\left\{\mathcal{C}_{\mathrm{S}^{\prime}}, G\right\}
$$

Here the -1 denotes the inverse of the given matrix whose $\mathbf{S}$ indices index irreducibly [56, 88] second-class constraints. (Irreducibly here refers to these constraints not being combineable in any manner so as to separate out any further functionally-independent first-class constraints.) Then the classical brackets role played ab initio by the Poisson brackets gets taken over by the Dirac brackets. Moreover, e.g. [88, 146] exposit how Dirac brackets can be viewed geometrically as a more reduced formulation's version of Poisson brackets. The particular Dirac brackets formed once no second-class constraints remain illustrates the concept of 'final classical brackets' forming a 'final classical brackets algebra' of constraints. (This is in contrast with naïve Poisson brackets as an 'incipient' notion of bracket.) First-class constraints use up 2 degrees of freedom each; second-class, only 1.

Some constraints are regarded as gauge constraints; however exactly which constraints these comprise is disputed. What is agreed upon is that second-class constraints are not gauge constraints; all gauge constraints use up two degrees of freedom. Dirac [56] conjectured a forteriori that all first-class constraints are gauge constraints ${ }^{5}$, so using up 2 degrees of freedom would then conversely imply being a gauge constraint. However Section 2 outlines how this conjecture has been refuted, alongside various other perspectives on the status of gauge constraints.

Gauge-fixing conditions $\mathrm{F}_{\mathrm{X}}$ may then be applied to whatever gauge theory (though one requires the final answers to physical questions to be gauge-invariant). These are a means of removing gauge freedom by fixing a choice of gauge, though physical answers are required to end up in gauge-invariant form.

As a final remark, second-class constraints can always in principle ${ }^{6}$ be handled by alternatively thinking of them as 'already-applied' gauge fixing conditions that can be recast as first-class constraints by adding suitable auxiliary variables to one's configuration space or phase space. By doing this, a system with first- and second-class constraints can be turned into a more redundant description of a system with just first-class constraints. Sets of first-class constraints obtained in this way are known as effective constraints [33].

\subsection{Examples of constraints in theoretical physics}

Most of the theories given here are used as recurring examples in this review; using multiple examples in reviews is in the tradition of Isham [93] and Kuchař [106]. I enumerate the example theories and models in this review with fixed example numbers 0 to 10 to keep these recurrences manifest.

Example 1. Electromagnetism in vacuo has the ${ }^{7}$

$$
\text { (Gauss constraint) } \quad \mathcal{G}:=\partial_{i} \pi^{i}=0 .
$$

\footnotetext{
${ }^{5}$ This is in Dirac's sense of 'gauge constraint' as per Section 1.3.

${ }^{6}$ To [88]'s precursor statement, I add the caveat 'locally', because gauge-fixing conditions themselves in general are not global entities.

${ }^{7}$ Some notation for this subsection is as follows. I use capital Latin indices for particle labels or internal indices, depending on context. $A_{i}$ is the electromagnetic vector potential with conjugate momentum $\pi^{i}$. $q^{I}$ are particle positions with conjugate momenta $p_{I}$ and masses $m_{I}$. The 4 - $d$ spacetime is the pair $\left(\mathfrak{m}, g_{\mu \nu}\right)$. Here $\mathfrak{m}$ is the spacetime topological manifold and $g_{\mu \nu}^{-}$is a metric that provides this with semi-Riemannian geometrical structure. $g_{\mu \nu}=g_{\mu \nu}\left(X^{\rho}\right)$, for $X^{\rho}$ spacetime coordinates. The $3-d$ spaces are pairs $\left(\Sigma, h_{i j}\right)$ for a fixed topological manifold $\Sigma$. Thus such dynamical study restricts $\mathfrak{m}$ to be of the simple form $\Sigma \times I$ for $I$ some kind of interval in $\mathbb{R}$. Moreover, this fixed spatial topological space is taken in this review to be a compact without boundary one. Finally $\Sigma$ additionally comes equipped with suitable differential and metric structure. $h_{i j}=h_{i j}\left(x^{k}\right)$, for $x^{k}$ spatial coordinates, is a spatial metric, with determinant $h$, covariant derivative $D_{i}$, Ricci scalar $R=R\left(x^{e} ; h_{f g}\right]$, and conjugate momenta $p^{i j}$ with trace $p$.
} 
This arises from variation with respect to the electromagnetic potential $\Phi$. One also has $\pi_{\Phi}=0$, for $\pi_{\Phi}$ the momentum conjugate to $\Phi$. These are both first-class, and use up 2 degrees of freedom each, so one passes from $A_{i}, \Phi$ and their conjugate momenta's redundant $4 \times 2$ phase space degrees of freedom per space point to just $2 \times 2$. This is in accord with electromagnetic waves consisting of just 2 transverse modes. This $\mathcal{G}$ is uncontroversially a gauge constraint, associated with the U(1) group. Further Gauss constraints that share these conceptual properties feature in many other theories. Examples of such are in 1) pure Yang-Mills theory (with internal index $I: \mathcal{G}_{I}$ ), 2 ) the scalar and fermionic gauge theories that one can associate with each of electromagnetism and Yang-Mills theory. (See [167] for more details of 1) and 2)).

Example 2. Barbour-Bertotti's [7, 28] scaled relational particle mechanics has the

$$
\begin{aligned}
& \text { (zero total momentum constraint) } \quad \underline{\mathcal{P}}:=\sum_{I=1}^{N} \underline{p}_{I}=0, \quad \text { and } \\
& \text { (zero total angular momentum constraint) } \quad \underline{\mathcal{L}}:=\sum_{I=1}^{N} \underline{q}^{I} \times \underline{p}_{I}=0 .
\end{aligned}
$$

Here $I$ runs over particle labels 1 to $N$. These constraints from variation with respect to some translational and rotational auxiliary variables respectively; relatedly, these constraints generate the Euclidean group of translations and rotations. They are first-class and use up 2 degrees of freedom per constraint degree of freedom. Thus one passes from a redundant configuration space $\mathbb{R}^{N d}$ (in dimension $d$ ) to a reduced configuration space $\mathbb{R}^{N d} / \operatorname{Eucl}(d)$. This amounts to removing Newton's absolute space from mechanics. Note that these are again not internal gauge constraints, but they are uncontroversially gauge constraints once more. Molecular physics has similar classical kinematics in its zero angular momentum case; the nonzero angular momentum case, however, has a more complicated fibre bundle structure (consult [113] if interested in this difference).

Example 3. Arnowitt-Deser-Misner (ADM)'s geometrodynamical formulation of GR (Fig. 1) involves the

$$
\text { (momentum constraint) } \quad \mathcal{M}_{i}:=-2 D_{j} p^{j}{ }_{i}=0 .
$$

This arises from variation with respect to ADM's shift $N^{i} . \mathcal{M}_{i}$ is first-class, and uses up 2 degrees of freedom per space point. It is also uncontroversially a gauge constraint, with the spatial diffeomorphisms $\operatorname{Diff}(\Sigma)$ as the corresponding gauge group.

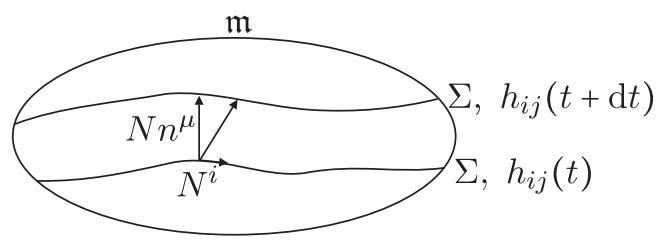

Figure 1. ADM split of spacetime $\mathfrak{m}$ with respect to spatial hypersurfaces $\Sigma$. $n^{\mu}$ is the normal to the hypersurface, $N$ is the lapse (time elapsed) and $N^{i}$ is the shift (point identification map). Together, these form a strutting: how to fit together adjacent hypersurfaces within spacetime. For later use, 1) the normal to the spatial hypersurface $\Sigma$ then takes the computational form $n^{\mu}=\left[N^{-1},-N^{-1} N^{i}\right]$. 2) $N^{\mu}:=\left[N, N^{i}\right]$ is the spacetime 4 -vector of auxiliaries, with conjugate momenta $P_{\mu}$.

The feature of using up degrees of freedom in pairs also applies to the GR

$$
\text { (Hamiltonian constraint) } \quad \mathcal{H}:=\left\{p_{i j} p^{i j}-p^{2} / 2\right\} / \sqrt{h}-\sqrt{h} R=0 .
$$

In the ADM formulation of GR this also arises as a secondary constraint from variation with respect to the lapse $N$. On the other hand, in the Baierlein-Sharp-Wheeler or related forms 
of GR [23, 25], $\mathcal{H}$ arises rather as a primary constraint corresponding to the action being reparametrization invariant along the lines of (1.3). The same is true for relational particle mechanics'

$$
\text { (energy constraint) } \quad \mathcal{E}:=\sum_{I=1}^{N} p_{I}^{2} / 2 m_{I}+V\left(\underline{q}^{I}\right)=E,
$$

as argued below ( $V$ is here the potential function). Thus the below two examples illustrate that the primary-secondary distinction is artificial in that it is malleable by change of formalism. Thus we will avoid that distinction in this review other than possibly pointing out others' claims that concern it (and counterexamples).

Example 2 relational particle mechanics' energy constraint $\mathcal{E}$ arises as a primary constraint from their Jacobi-type action $[7,28,110]^{8}$

$$
\begin{aligned}
& \mathrm{S}=\int \operatorname{Ld} \lambda=2 \int \sqrt{T\{E-V\}} \mathrm{d} \lambda, \\
& T=\delta_{I J} m_{I}\left\{\underline{\dot{q}}^{I}-\underline{\dot{a}}-\left\{\underline{\dot{b}} \times \underline{q}^{I}\right\}\right\}\left\{\underline{\dot{q}}^{J}-\underline{\dot{a}}-\left\{\underline{\dot{b}} \times \underline{q}^{J}\right\}\right\} / 2 .
\end{aligned}
$$

Moreover in this case the way the purely quadratic form of the Lagrangian causes the constraint to arise is in close analogy to Pythagoras' theorem/direction cosines summing to one.

Example 4. Then the Baierlein-Sharp-Wheeler or related formulations [23, 25] of GR have the GR Hamiltonian constraint $\mathcal{H}$ arise as one primary constraint per space point in close analogy to Example 4 working.

\subsection{Interlude: notions of gauge theory}

One conceptually useful way of introducing gauge theory ${ }^{9}$ is by letting $\mathfrak{g}$ be a group of transformations held to be physically redundant that acts on $\mathfrak{q}$ (or sometimes, when specified in this review, on phase space). This group ('gauge group'), the constraints and gauge theory can then be inter-related as follows.

1) The mathematically-disjoint auxiliaries $g^{\mathrm{G}}$ are $\mathfrak{g}$-auxiliaries that encode the group action of $\mathfrak{g}$. (Mathematically-disjoint means like $\Phi$ not being part of a larger tensorial package in the sense that the longitudinal piece of $A^{a}$ is part of the larger entity $A^{a}$ itself.) Then at least in the set of examples given above, first-class secondary constraints arise from variation with respect to mathematically-disjoint auxiliary variables. Furthermore, the effect of this variation is to additionally use up part of an accompanying mathematically coherent block that however only contains partially physical information; this is clear in the above discussions of electromagnetism.

2) The disjoint auxiliary variables are moreover often in correspondence with a group of redundancies $\mathfrak{g}$. Variation with respect to the mathematically disjoint auxiliary variable $g^{\mathrm{G}}$ produces the gauge constraints, denoted $\mathcal{G} \mathcal{A} \mathcal{U} \mathcal{G} \mathcal{E}_{\mathrm{G}}$ for clarity. We then do not need to isolate the latter for many purposes: E.g. in the case of electromagnetism, the Gauss constraint $\mathcal{G}$ associated with this pair already arises from varying with respect to the auxiliary $\Phi$. It is much more convenient to obtain $\mathcal{G}$ in this way because $\Phi$ is a mathematically isolated object and so one can entirely straightforwardly vary with respect to it. This is one reason why the most habitual and sometimes the only possible - redundant formulations of gauge theories are useful to work with. Some theoreticians (contrast with Section 2.1) preclude from 2) the reparametrization and refoliation groups on grounds that they are dynamically distinct. The included groups,

\footnotetext{
${ }^{8}$ Here $\underline{a}$ and $\underline{b}$ are translational and rotational auxiliaries respectively. $m_{I}$ are particle masses, $E$ is the total energy and $T$ is the kinetic term.

${ }^{9}$ We take this to have a wider meaning than just the typical gauge theories of particle physics. It covers also e.g. the gauge theories in molecular physics [113], relational particle mechanics [7, 28], cosmological perturbation theory [30, 117], and those associated with various kinds of diffeomorphisms (see e.g. [40, 126]).
} 
the constraints corresponding to which are first-class and linear - denoted $\mathcal{L} \mathcal{I} \mathcal{N}_{\mathrm{L}}$ - fall under Barbour's best matching paradigm (outlined in Appendix A) and correspond to redundancies in the instantaneous configurations ${ }^{10}$.

The precluded constraints are quadratic constraints (corresponding to strutting 'orthogonal' to the instantaneous configurations: see Fig. 1). Ways in which refoliation is more subtle than reparametrization are outlined in Section 1.7. In the commonly-encountered physical theories, the diagnostic for the precluded constraints is that they have quadratic and not linear dependence in the constraints; hence I denote these by $\mathcal{Q U} \mathcal{A D}$.

Note that naming a $\mathfrak{g}$ as a candidate gauge group can be a formalism-dependent rather than theory-dependent statement. On the one hand, it is at least in principle possible to rewrite a theory possessing a gauge freedom in terms of true dynamical degrees of freedom alone (see also Section 3.1). (This 'in principle' does not imply that the equations one would need to solve to do so can be solved.) On the other hand, variable numbers of auxiliary degrees of freedom can be added, and some such cause different gauge constraints to appear or to recast existing non-gauge constraints as gauge ones. These considerations parallel those for removing second-class constraints in Section 1.1. Moreover notions 1) and 2) can be applied, for a subset of theories, to the subset of constraints that are linear. I.e. that subset of theories for which $\mathcal{Q U} \mathcal{A D}$ (or any other nonlinear constraint) is not an integrability of the linear constraints $\mathcal{L I N}_{\mathrm{L}}$ (see Section 1.7 for a counterexample). Thus here $\mathcal{L} \mathcal{I} \mathcal{N}_{\mathrm{L}}$ constitutes a subalgebraic structure (taken to cover both subalgebras and subalgebroids, see Section 1.7) of constraints.

There are of course further conceptualizations of gauge and of gauge theory [41, 138, 166, 167]. For instance, one can do so in terms of the presence of free functions in the solution of the equations of motion or of making global symmetries local.

A further, older distinction between different uses of the word 'gauge' concerns what a theory is a gauge theory of, e.g., $Q^{\mathrm{A}}$ alone, $Q^{\mathrm{A}}$ and $P_{\mathrm{A}}$, whole paths, or histories. Bergmann's early position [38] was that that gauge theory concerns whole paths (dynamical trajectories): path-gauge notion. This is in contrast to Dirac's perspective $[55,56]$ that gauge theory concerns data at a given time: data-gauge notion (called ' $\mathrm{D}$ ' by Bergmann and Komar [39], albeit that stood for 'Dirac' rather than for 'data') ${ }^{11}$. I.e. gauge group action in spacetime versus spatial/configurational/canonical settings.

\subsection{More examples of constraints toward quantum gravitational theories}

Example 5. Ashtekar Variables formulation of GR's constraints are ${ }^{12}$

$$
\mathcal{G}_{I}:=D_{i} E_{I}^{i}=0, \quad \mathcal{M}_{i}:=E^{j I} F_{j i I}=0, \quad \mathcal{H}:=\epsilon_{I J K} E^{i I} E^{j J} F_{i j}^{K}=0 .
$$

Example 6. Proca theory (the massive counterpart of electromagnetism) is a simple example of a theory with a second-class constraint [124]

$$
\mathcal{C}:=\partial_{i} \pi^{i}+m^{2} \Phi=0
$$

\footnotetext{
${ }^{10}$ Thus the corresponding notion of gauge is instantaneous, i.e. along the lines of Dirac's notion and not Bergmann's, as discussed below.

${ }^{11}$ N.B. path-gauge and data-gauge are definitions of notions of gauge, as opposed to particular choices of gauge within a particular notion of gauge such as Coulomb gauge or Lorenz gauge for electromagnetism). In this review, I also take 'history' to mean more than just a dynamical path; at the quantum level these are paths that are furthermore decorated with projection operators. Also N.B. that this review's namings are preferentially based on each entity's conceptual content - true name - rather than on e.g. the name of who discovered it, or on how the entity was once thought about prior to developments in its conceptual understanding. Of course, upon first introduction of such terms, I give what aliases they are or have been known by.

${ }^{12}$ I present just the complex case for simplicity. $E_{I}^{i}$ is the $\mathrm{SU}(2)$ equivalent of electric field flux. $F_{i j I}$ is the corresponding field strength. $E_{I}^{i}$ is now also geometrically a particular kind of bein ('square root' of the spatial metric configuration variable), and yet has been recast as a momentum variable by canonical transformation.
} 
This indeed uses up only 1 degree of freedom, so this theory has 1 more physical mode than electromagnetism itself. Gravitational theories with second-class constraints include 1) EinsteinCartan theory [101], 2) Einstein-Dirac theory (i.e. GR with spin-1/2 fermion matter as required for a full model of the known fields of nature) [52], 3) supergravity [52, 53, 64, 124, 154] (on the one hand supersymmetric particles are being sought for at the LHC, and on the other hand this review reveals a number of further ways in which supergravity differs from GR).

Example 7. Locally-Lorentz constraints in first-order formulations have constraints $\mathcal{J}_{A B}$ (and conjugate; the capital indices here are specifically 2-spinor indices). These occur in e.g. in Einstein-Dirac theory and supergravity.

Example 8. Supersymmetric constraints, a particular case of which in gravitational theories are supergravity's constraint $\mathcal{S}_{A}$ (and conjugate).

See e.g. [52] for explicit forms for $\mathcal{J}_{A B}$ and $\mathcal{S}_{A}$ (these details are not required for this review, which only makes use of the form of the algebraic structure of the constraints).

\subsection{Different kinds of notions of equality in the principles of dynamics}

We already explained weak equality in Section 1.1: 'up to functionals of the first-class constraints'. 'Strong equality' means equality in the usual sense ${ }^{13}$. E.g. Isham [93] points out the possibility of making the strong equality demand in defining notions of beables. Finally, Batalin and Tyutin [33] consider equality up to effective constraints, denoted by the symbol $\approx$. I term this effective weak equality. Moreover, one person's effective formulation could have been written down ab initio by another as a formulation happening to have no second-class constraints, so this is not so large a distinction.

\subsection{Different kinds of brackets}

We have already encountered the Poisson brackets and the Dirac brackets in Section 1.1. A distinct generalization of the Poisson bracket - to mixtures of bosonic and fermionic species - is Poisson bracket here generalizes to the Casalbuoni brackets [50]

$$
\{F, G\}_{\mathbf{C}}:=\frac{\partial F}{\partial Q^{\mathrm{A}}} \frac{\partial G}{\partial P_{\mathrm{A}}}-(-)^{\epsilon_{F} \epsilon_{G}} \frac{\partial G}{\partial Q^{\mathrm{A}}} \frac{\partial F}{\partial P_{\mathrm{A}}} .
$$

Here $\epsilon_{\mathrm{A}}$ is the Grassmann parity of species A: 1 for bosons and -1 for fermions. This also readily generalizes to field-theoretic form. It obeys the Grassmannian generalization of the Jacobi identity,

$$
\left\{\{F, G\}_{\mathbf{C}}, H\right\}_{\mathbf{C}}(-1)^{\epsilon_{F} \epsilon_{H}}+\text { cycles }=0 .
$$

The quantum commutator counterpart of the above types of brackets is covered in Section 1.9. See Section 7 for yet further types of classical and quantum brackets.

\subsection{Algebraic structures resulting from the introduction of brackets}

Given a type of bracket, there is the additional issue of mathematical type of the algebraic structure formed by entering the theory's constraints into that type of brackets. It is well known that if the right hand side is of the form of a sum of (structure constants) $\times$ constraints, the brackets of constraints constitute a Lie algebra. However, if instead structure functions materialize, one has a more general structure termed an algebroid [42, 46, 160]. This clearly

\footnotetext{
${ }^{13}$ E.g. [88] give a form for this as a linear combination of constraints. I however retain the general definition for its subsequent use in studying global effects.
} 
structurally precedes the notions of beables and of beables algebraic structures, through using a strict subset of the structures that this requires.

Example 1. Gauss constraints form Lie algebras. These can be Abelian (electromagnetism $)^{14}$

$$
\{(\mathcal{G} \mid \chi),(\mathcal{G} \mid \zeta)\}=0
$$

or non-Abelian (Yang-Mills theory)

$$
\left\{\left(\mathcal{G}_{I} \mid \chi^{I}\right),\left(\mathcal{G}_{J} \mid \zeta^{J}\right)\right\}=f_{I J}^{K}\left(\mathcal{G}_{K} \mid \chi^{I} \zeta^{J}\right)
$$

for Lie algebra structure constants $f_{I J}{ }^{K}$.

Example 2. 3-d relational particle mechanics has

$$
\left\{\mathcal{P}_{i}, \mathcal{P}_{j}\right\}=0, \quad\left\{\mathcal{L}_{i}, \mathcal{L}_{j}\right\}=\epsilon_{i j}{ }^{k} \mathcal{L}_{k}, \quad\left\{\mathcal{P}_{i}, \mathcal{L}_{j}\right\}=\epsilon_{i j}{ }^{k} \mathcal{P}_{k} .
$$

The meanings of these are, respectively, that the $\mathcal{L}_{i}$ form an $\mathrm{SO}(3)$ subalgebra of rotations, the $\mathcal{P}_{i}$ form an Abelian subalgebra of translations, and $\mathcal{P}_{i}$ is a good $\mathcal{L}_{i}$ vector. All other Poisson brackets for this model are zero.

Example 3 or 4 . Spatial diffeomorphisms by themselves form an infinite-dimensional Lie algebra,

$$
\left\{\left(\mathcal{M}_{i} \mid L^{i}\right),\left(\mathcal{M}_{j} \mid M^{j}\right)\right\}=\left(\mathcal{M}_{k}|[L, M]|^{k}\right) .
$$

Here $L_{i}(z)$ and $M_{i}(z)$ are vectorial smearing functions and |[, ]| is the standard Lie bracket.

Example 3 or 4 . In the case of full GR as geometrodynamics, the constraints' Poisson brackets are [56] (1.4),

$$
\begin{aligned}
& \left\{(\mathcal{H} \mid K),\left(\mathcal{M}_{i} \mid L^{i}\right)\right\}=\left(£_{L} \mathcal{H} \mid K\right), \\
& \{(\mathcal{H} \mid J),(\mathcal{H} \mid K)\}=\left(\mathcal{M}_{i} h^{i j} \mid J \overleftrightarrow{\partial}_{i} K\right) .
\end{aligned}
$$

Here $J(z), K(z)$ are scalar smearing functions, $£$ is the well-known Lie derivative of differential geometry [147], and $X \overleftrightarrow{\partial}_{i} Y:=\left(\partial_{i} Y\right) X-Y \partial_{i} X$ (a notation familiar from QFT).

(1.5) means that $\mathcal{H}$ is a 'good object' (a scalar density) under spatial diffeomorphisms $\operatorname{Diff}(\Sigma)$. (1.6), however, is more complicated in both form and meaning. Firstly, it uncontroversially means that the linear $\mathcal{M}_{i}$ arises as an integrability of the quadratic $\mathcal{H}$. Secondly, the presence of $h^{i j}\left(h_{k l}\right)$ on its right-hand side is a structure function. Thus this bracket renders the overall algebraic structure more complicated than a Lie algebra. It is, rather, an algebroid: specifically the Dirac algebroid [56, 42]. This is entirely unlike the unsplit GR's spacetime diffeomorphisms $\operatorname{Diff}(\mathfrak{m})$ which form a genuine Lie algebra paralleling that of $\operatorname{Diff}(\Sigma)$.

$\mathcal{H}$ 's distinction from $G R$ theories' linear constraints has further fuel than $\mathcal{E}$ 's from relational particle mechanics linear constraints, as follows. i) Refoliation invariance is a hidden invariance. (This is as opposed to an invariance that is manifest in the canonical formulation itself. In particular, one needs to foliate spacetime in order to have a canonical formulation, and one cannot directly see refoliation invariance within any particular foliation.) ii) (1.6) implies that GR's linear momentum constraint $\mathcal{M}_{i}$ is an integrability condition that follows from the existence of $\mathcal{H}$. Thus it ceases to be possible to consider $\mathcal{Q U} \mathcal{A D}$ and $\mathcal{L} \mathcal{I} \mathcal{N}_{\mathrm{L}}$ piecemeal in generallyrelativistic theories. iii) It is specifically the presence of $\mathcal{H}$ that causes the algebraic structure of these constraints to be an algebroid. Structure functions are needed to accommodate the

\footnotetext{
${ }^{14}$ This is given with smearing functions $\chi(z)$ and $\zeta(z)$. More generally, $\left(\mathcal{C}_{\mathrm{Z}} \mid A^{\mathrm{Z}}\right):=\int d^{3} z \mathcal{C}_{\mathrm{Z}}\left(z^{i} ; h_{j k}\right] A^{\mathrm{Z}}\left(z^{i}\right)$ denotes an 'inner product' notation for the smearing of a Z-tensor density valued constraint $\mathcal{C}_{\mathrm{Z}}$ by an oppositerank Z-tensor smearing with no density weighting, $A^{\mathrm{Z}}$.
} 
variety of possible foliations; see e.g. [95, 96, 112, 153] for further discussions of the 'group action' involved.

Example 5. The Ashtekar variables' algebraic structure of constraints $[18,155]$ is much like geometrodynamics', but with an extra Gauss-type constraint included. This further includes a bracket between two SU(2) Yang-Mills-Gauss constraints (these simply commute with the two other constraints). It is the bracket of two $\mathcal{H}$ 's that continues to cause difficulty, and for reasons unchanged from the geometrodynamical case's.

Examples 6 and 7. See e.g. [52, 161] for what is known about the Einstein-Dirac and supergravity constraint algebras. In particular, supergravity is an example of a subset of the linear constraints - the supersymmetry constraints - having the supergravity counterpart of the quadratic $\mathcal{H}$ as their integrability condition. This follows from [52, 154]

$$
\left\{\left(\mathcal{S}_{A} \mid \theta^{A}\right),\left(\overline{\mathcal{S}}_{A^{\prime}} \mid \bar{\theta}^{A^{\prime}}\right)\right\}_{\mathbf{C}}^{*} \propto i\left(\gamma^{A A^{\prime}} \mid \mathcal{H}_{A A^{\prime}}\right)+\text { terms with } \mathcal{J}_{A B} \text { or its conjugate as a factor.(1.7) }
$$

Here $\mathcal{H}_{A A^{\prime}}:=n_{A A^{\prime}} \mathcal{H}_{\perp}+e_{A A^{\prime}}{ }^{i} \mathcal{M}_{i}$ packages together the supergravity Hamiltonian and momentum constraints using the normal $n$ and spinor-valued 1-form $e$. (Less importantly, $\theta^{A}$ and $\bar{\theta}^{A^{\prime}}$ are fermionic smearing functions, whereas $\gamma^{A A^{\prime}}\left(\theta^{B}, \bar{\theta}^{B^{\prime}}\right)$ is some composite of these that is itself another smearing function.) (1.7) is the basis for a number of significant new results in this review.

(1.7) can furthermore be interpreted [154] in terms of $\mathcal{S}_{A}$ being a square root of $\mathcal{H}$ in parallel to how the Dirac operator is well known to be a square root of the Klein-Gordon one. This may provide reasons why $\mathcal{H}$ is, after all, not so fundamental. However, it should be cautioned that whereas Dirac's corresponding fermions were observationally vindicated, this is not the case to date as regards superpartner particles. This can be taken as a limitation on arguing against the fundamentality of quadratic constraints like $\mathcal{H}$ on the grounds of their being supplanted in supersymmetric theories.

To sum up, schematically, for a theory with constraints, the constraint algebra is

$$
\left|\left[\mathcal{C}_{\mathrm{F}}, \mathcal{C}_{\mathrm{F}^{\prime}}\right]\right| \approx 0 .
$$

Indexing these constraints by F's indicates that they are first-class. Any second-class ones there were have been removed by one of the following. a) Extension, in which case it indeed involves a Poisson bracket, effective E-index and effective weak equality symbol $\approx$. b) Passing to the Dirac bracket whilst leaving the F-index and weak equality symbol untouched.

\subsection{Classical notions of beables}

Finally, a theory with constraints and brackets possesses a further class of conceptually important objects: those that form (usually) weakly zero brackets with the constraints,

$$
\left|\left[\mathcal{C}_{\mathrm{F}}, B_{\mathrm{B}}\right]\right| \approx 0 \text {. }
$$

Comparing (1.8) and (1.9) implies that the $\mathcal{C}_{F}$ themselves are in some sense beables. However, already $\mathcal{C}_{\mathrm{F}} \approx 0$, so we are really looking for further quantities that are not trivial in this way. Let us call these other quantities proper beables; the rest of the article will always mean 'proper beables' whenever it says 'beables'. Together, (1.8), (1.9) and closure of beables carry no nontrivial algebraic structure at the level of weak equality than just the closure of beables. This is because they are just the conditions for a direct product with the weakly-Abelian constraint algebra.

In all cases beables themselves are to close as an algebraic structure under the same type of bracket that they are defined by. E.g. if $B_{\mathrm{B}}$ are beables in the sense of $(1.1)$, then $\left|\left[B_{\mathrm{B}}, B_{\mathrm{B}^{\prime}}\right]\right|$ 
are too. I.e. this bracket object itself obeys property (1.1). This is by two uses of (1.1) in the Jacobi identity with two $B$ 's and one $\mathcal{C}$ :

$$
\left|\left[\mathcal{C}_{\mathrm{F}},\left|\left[B_{\mathrm{B}}, B_{\mathrm{B}^{\prime}}\right]\right|\right]\right|=-\left|\left[B_{\mathrm{B}},\left|\left[B_{\mathrm{B}^{\prime}}, \mathcal{C}_{\mathrm{F}}\right]\right|\right]\right|-\left|\left[B_{\mathrm{B}^{\prime}},\left|\left[\mathcal{C}_{\mathrm{F}}, B_{\mathrm{B}}\right]\right|\right]\right|=0
$$

On the other hand, two uses of (1.1) in the less usual Jacobi identity with two $\mathcal{C}$ 's and one $B$,

$$
\left|\left[B_{\mathrm{B}},\left|\left[\mathcal{C}_{\mathrm{F}}, \mathcal{C}_{\mathrm{C}^{\prime}}\right]\right|\right]\right|=-\left|\left[\mathcal{C}_{\mathrm{F}},\left|\left[\mathcal{C}_{\mathrm{F}^{\prime}}, B_{\mathrm{B}}\right]\right|\right]\right|-\left|\left[\mathcal{C}_{\mathrm{F}^{\prime}},\left|\left[B_{\mathrm{B}}, \mathcal{C}_{\mathrm{F}}\right]\right|\right]\right|=0
$$

enforces the following. A particular notion of beables $B_{\mathrm{B}}$ corresponding to forming zero brackets with a subset of a theory's constraints $\mathcal{C}_{\mathrm{C}}$ is only self-consistent if $B_{\mathrm{B}}$ also forms zero brackets with $\left|\left[\mathcal{C}_{\mathrm{F}}, \mathcal{C}_{\mathrm{F}^{\prime}}\right]\right|$. I.e. with the algebraic closure of that subset of constraints. Thus one can only consistently adopt subsets of constraints that are furthermore subalgebraic structures with respect to the given brackets.

Different notions of beables then concern different subalgebraic structures of constraints.

Classical Dirac beables (Section 3) are functionals $D_{\mathrm{D}}=\mathrm{F}_{\mathrm{D}}\left[Q^{\mathrm{A}}, P_{\mathrm{A}}\right]$ that classical-bracketcommute with all of a theory's first-class constraints $\mathcal{C}_{\mathrm{F}}$ then using the Poisson, Dirac or the enlarged bracket for the purpose of assigning beables

$$
\left\{\mathcal{C}_{\mathrm{F}}, D_{\mathrm{D}}\right\} \approx 0
$$

is the standard form for this. Batalin and Tyutin also introduced a weak effective equality version [33]. In the case in which time evolution is generated by a constraint, Dirac beables are also known as constants of the motion alias perennials [29, 37, 62, 68, 75, 76, 107, 109, 169]. True $[132,133,134]$ alias complete observables/beables $[135,136]$ (which at least [155] also terms evolving constant of the motion) are also a notion of this kind. They involve operations on a system each of which produces a number that can be predicted if the state of the system is known. See Section 3 for examples.

Note 1. If there were second-class constraints, we would pass to Dirac or extended brackets, whence they are absent and then define Dirac beables as before but in terms of this new bracket.

Note 2. The name 'constants of the motion' conventionally follows from a generally-covariant (at least in Henneaux and Teitelboim's sense [88]) theory's total Hamiltonian being of the form $\mathrm{H}=\int_{\Sigma} \mathrm{d} \Sigma \Lambda^{\mathrm{F}} \mathcal{C}_{\mathrm{F}}$ for multiplier coordinates $\Lambda^{\mathrm{F}}$. Then

$$
\mathrm{d} D_{\mathrm{D}} / \mathrm{d} t=\left\{D_{\mathrm{D}}, \mathrm{H}\right\}=\left\{D_{\mathrm{D}}, \int_{\Sigma} \mathrm{d} \Sigma \Lambda_{\mathrm{F}} \mathcal{C}^{\mathrm{F}}\right\}=\int_{\Sigma} \mathrm{d} \Sigma \Lambda_{\mathrm{F}}\left\{D_{\mathrm{D}}, \mathcal{C}^{\mathrm{F}}\right\} \approx 0
$$

with the last equality following from (1.12). Thus it would appear that 'nothing happens' (a type of frozen argument), though Section 5.4 attributes this to a fallacy.

Note 3. 't Hooft [150] used a notion of 'beables' that are conceptually disjoint from his notion of 'changeables'; as a frozen notion, however, 't Hooft's notion of beables is more stringent than the notion of beables used in this review.

On the other hand, classical Kuchař beables [29, 37, 62, 103, 107, 109, 169] (Section 2) are functionals $K_{\mathrm{K}}=\mathrm{F}_{\mathrm{K}}\left[Q^{\mathrm{A}}, P_{\mathrm{A}}\right]$ that classical-brackets-commute with all linear constraints

$$
\left\{K_{\mathrm{K}}, \mathcal{L} \mathcal{I} \mathcal{N}_{\mathrm{L}}\right\} \approx 0 .
$$

Kuchař beables are more straightforward to construct; see Section 2 for examples. These correspond to an uncontroversial if perhaps somewhat restrictive notion of gauge invariance. Namely the one given in Section 1.3 in terms of the gauge group $\mathfrak{g}$ that corresponds to the linear constraints $\mathcal{L I N}_{\mathrm{L}}$. Kuchař beables are then gauge-invariant quantities in the 'Dirac' sense familiar from electromagnetism and the canonical formulation of particle physics. Using Kuchař beables reflects treating $\mathcal{Q U} \mathcal{A D}$ distinctly from $\mathcal{L I N}_{\mathrm{L}}$; see Section 2 for further motivation for this. 
N.B. that weak and effective-weak notions are tied to the uncontroversial notion of first-class constraints rather than to gauge constraints. In the case of standard canonical formulations GR, effective is equivalent to first-class, and so effective-weak is equivalent to weak.

Classical partial observables are a point of view that began with Rovelli's works [47, 48, $132,133,134]$ though one might view $[54,121]$ as forerunners in some respects. See also [57, $58,135,138]$ and the reviews [137, 152, 155]. Partial observables do not require commutation with any constraints. Partial observables involve classical or QM operations on the system that produces a number that is measurable but possibly totally unpredictable even if the state is perfectly known (contrast with the definition of total/Dirac observables). The physics then lies in considering pairs of these objects, with correlations between them encoding extractable purely physical information. I.e. correlations of two partial observables are predictable; in particular the value of a partial observable A subject to another partial observable B taking a particular value is predictable, in which case partial observable B is playing a 'clock' role. It is not however clear exactly which partial observables correspond to realistic and accurate clocks. Nor is it clear how a number of other facets of the problem of time can be addressed via these [4, 7, 93, 106, 107]. What is clear is that the partial observables approach's correlations are themselves functions on the constraint surface and commute with the constraints; as such they furnish complete or Dirac observables/beables, according to one's interpretation.

Section 4 then covers local versus global notions of beables, and Section 5 covers Pons et al. diffeomorphism-specific work [98, 127, 128, 129, 130, 131]. The latter also covers how Bergmann observables/beables follow from his and various collaborators' position on the notion of gauge $[16,38,39]$.

\subsection{Quantum notions of beables}

The quantum versions of the definitions of beables (see Section 6 for more detail) involve selfadjoint operators that form zero quantum commutators with the quantum constraints

$$
[\widehat{A}, \widehat{B}]=\widehat{A} \widehat{B}-\widehat{B} \widehat{A}
$$

quantum Dirac beables are: $\widehat{D}_{\mathrm{D}}$ such that $\left[\widehat{D}_{\mathrm{D}}, \widehat{\mathcal{C}}_{\mathrm{F}}\right] \Psi=0$,

quantum Kuchař beables are: $\widehat{K}_{\mathrm{K}}$ such that $\left[\widehat{K}_{\mathrm{K}}, \widehat{\mathcal{L I N}}_{\mathrm{L}}\right] \Psi=0$.

Objects $\widehat{S}_{\mathrm{M}}$ obeying $\left[\widehat{\mathcal{Q U \mathcal { A D }}}, \widehat{S}_{\mathrm{M}}\right] \Psi=0$ are conventionally termed $S$-matrix quantities, after the QM's scattering matrix for interaction processes. Furthermore, these do not carry backgrounddependence connotations due to corresponding to 'scattering processes' in configuration space rather than in space itself. Clearly then for quantum beables, Kuchař and $S$-matrix $\Rightarrow$ Dirac. Quantum partial observables are defined exactly as before too, though now 'produce a number' carries inherent probabilistic connotations.

\subsection{The problem of beables}

The problem of beables $[4,7,56,93,106,137]$ is but one facet of the problem of time $[4,5,7$, 93, 106] (see Appendix A for other facets of this). It concerns that in the Kuchař and especially Dirac conceptualizations, it is hard to construct a sufficiently large set of beables to describe physical theory, in particular for gravitational theory.

Strategies for dealing with the problem of beables include considering each of the Kuchar and Dirac positions on the nature of beables to be sufficient. Kuchař beables can also be viewed as a potentially useful halfway house in the construction of Dirac beables. Partial observables as a problem of observables/beables strategy is along the lines of this problem being held to be a misunderstanding of the true nature of observables/beables. Partial observables are, rather, entities that are measurable but unpredictable by themselves, predictions here involving rather 
correlations between more than one such. On the other hand, another strategy is to use partial observables as intermediates toward obtaining Dirac observables/beables.

\section{Kuchař beables}

\subsection{Further motivation for Kuchar̆ beables}

1) The Dirac conjecture (Section 1.1) is false by e.g. a technically constructed but not physically motivated counterexample given in [88]: $\mathrm{L}=\exp (y) \dot{x}^{2} / 2$ suffices, with its $p_{x}=0$ constraint being first-class but not associated with a gauge symmetry.

2) The conjecture is contested on further grounds by e.g. Kuchař [109] and Barbour-Foster [29]; this is furthermore directly at odds with [88]. I point out here that this discrepancy is due to [88] allowing for $t$-dependent canonical transformations, $\mathrm{Can}_{t}$. These map reparametrizationinvariant actions to non-reparametrization-invariant actions; $\mathcal{Q U} \mathcal{A D}$ is then also not an invariant form under $\mathrm{Can}_{t}$. On the other hand, one has to presume that $\mathrm{Can}_{t}$ are not licit in Barbour-type relational perspectives, in which space/configuration space and timelessness are primary. Here temporal relationalism (Appendix A) is implemented by reparametrization-invariant actions, and the principles of dynamics is reformulated to suit there being no primary notion of time. Consequently $\mathcal{Q U} \mathcal{A D}$ and $\mathcal{L} \mathcal{I} \mathcal{N}_{\mathrm{L}}$ are qualitatively different types of entities in this perspective.

3) Kuchař beables are, moreover, simpler to find than Dirac ones; Kuchař was motivated by this rather than 2).

4) A set of Kuchař beables can be extended to produce a set of Dirac beables (see Section 3.3).

One problem of beables strategy is that Kuchař beables are all [29, 37, 62, 103, 106, 107, 109, 169]. Finding Kuchař beables is uncontroversially a timeless pursuit through its not involving the quadratic Hamiltonian constraint. The downside is that a constraint of this kind remains as a frozen equation at the quantum level. Thus one has to concoct some kind of emergent-time or timeless scheme to deal with this (see Appendix A).

\subsection{Examples of Kuchař beables posed}

I denote a sufficient set of Kuchař beables to describe one's theory by $K_{\mathrm{K}}$.

Example 0. For theories with no linear constraints such as the Jacobi formulation of mechanics or Misner's minisuperspace [7], Kuchař beables are just any quantities (subject to a caveat and rephrasing in Section 4).

Example 2. Kuchař beables for scaled relational particle mechanics obey

$$
\left\{\mathcal{L}_{i}, K_{\mathrm{K}}\right\} \approx 0, \quad\left\{\mathcal{P}_{i}, K_{\mathrm{K}}\right\} \approx 0
$$

Also in the elsewise often simpler case of Example 2.b: pure-shape relational particle mechanics [7] (shapes are relative-angle and ratio of relative separation information)

$$
\left\{\mathcal{D}, K_{\mathrm{K}}\right\} \approx 0
$$

Here $\mathcal{D}:=\sum_{I=1}^{N} \underline{q}^{I} \cdot \underline{p}_{I}$ is the zero dilational momentum constraint.

Example 1. Kuchař beables for electromagnetism obey

$$
\left\{\mathcal{G}, K_{\mathrm{K}}\right\} \approx 0,
$$

and similarly for Yang-Mills theory and the gauge theories that can be associated with each of these. 
Example 3 or 4. For GR-as-geometrodynamics, Kuchař beables obey

$$
\left\{\mathcal{M}_{i}, K_{\mathrm{K}}\right\} \approx 0 \text {. }
$$

Example 5. For GR in Ashtekar variables, Kuchař beables obey

$$
\left\{\mathcal{M}_{i}, K_{\mathrm{K}}\right\} \approx 0, \quad\left\{\mathcal{G}_{I}, K_{\mathrm{K}}\right\} \approx 0
$$

Counter-example 7. Kuchař beables are not well-defined for supergravity. This is because the $\mathcal{S}_{A}$ 's algebraic primality over $\mathcal{H}$ - that the commutator of two $\mathcal{S}_{A}$ produces $\mathcal{H}(1.7)$ but not vice versa - means that supergravity's $\mathcal{L} \mathcal{I N}_{\mathrm{L}}$ do not form a subalgebraic structure of constraints. Then by the Casalbuoni brackets version of (1.11), a consistent notion of beables/observables cannot be associated with supergravity's $\mathcal{L I N}_{\mathrm{L}}$. Thus the notion of Kuchar beables is not available for supergravity, whether as a problem of beables resolution in itself or as a welldefined halfway house in the construction of Dirac beables.

\subsection{Kuchař beables examples resolved}

Example 0 is straightforward to resolve. Additionally, if linear constraints have been reduced out by whatever means, then one has arrived at a situation with equal status to Example 0. This is then the case for Examples 2 and 2.b. Pure-shape relational particle mechanics is simpler [6]: classical Kuchař beables are functionals of the shapes $\mathrm{S}^{\mathrm{A}}$ and their conjugate shape momenta $\mathrm{P}_{\mathrm{A}}^{\mathrm{S}}$,

$$
K_{\mathrm{K}}=\left\{\mathrm{F}_{\mathrm{K}}\left[\mathrm{S}^{\mathrm{A}}, \mathrm{P}_{\mathrm{A}}^{\mathrm{S}}\right]\right\} \text {. }
$$

For scaled relational particle mechanics, classical Kuchař beables are functionals of a scale variable $\sigma$, its conjugate scale (dilational) momentum $\mathrm{P}_{\sigma}$ and the shape and shape momenta,

$$
K_{\mathrm{K}}=\left\{\mathrm{F}_{\mathrm{K}}\left[\mathrm{S}^{\mathrm{A}}, \sigma, \mathrm{P}_{\mathrm{A}}^{\mathrm{S}}, \mathrm{P}_{\sigma}\right]\right\} \text {. }
$$

For example, for the scaled relational triangle [3], the shape space is the sphere. Using massweighted relative Jacobi vectors $\underline{\rho}_{1}, \underline{\rho}_{2}$ (Fig. 2a) convenient forms for the shapes are $\Theta=$ $2 \arctan \left(\rho_{2} / \rho_{1}\right)$ and $\Phi=\arccos \left(\underline{\rho}_{1} \cdot \underline{\rho}_{3} / \rho_{1} \rho_{3}\right)$. These are geometrically the spherical polar coordinates on the shape space sphere (Fig. $2 \mathrm{c})$. The scaled relational triangle also has a scale variable:

the moment of inertia, $I=\sum_{i=1}^{2} \rho_{i}^{2}$, or sometimes more conveniently its square root [7, 113]. The relational triangle's pure-shape momenta are then a relative angular momentum (between the base and the median) conjugate to $\Phi$ and a relative dilational momentum (dilatation of the base's length relative to the median's length) The relational triangle's scale momentum is an overall dilatation.

Shape and scale space is $\mathbb{R}^{3}$ topologically but not metrically (though it is conformally flat). The corresponding 'Cartesian' coordinates are the Hopf-Dragt coordinates [7, 113] (after the well-known Hopf map: $\left.\mathbb{S}^{3} \rightarrow \mathbb{S}^{2}\right)$ :

$$
\operatorname{Dra}_{1}:=2 \underline{\rho}_{1} \cdot \underline{\rho}_{2}, \quad \operatorname{Dra}_{2}:=2\left\{\underline{\rho}_{1} \times \underline{\rho}_{2}\right\}_{3}, \quad \operatorname{Dra}_{3}:=\rho_{2}^{2}-\rho_{1}^{2} .
$$

These and their conjugate momenta $\Pi_{i}^{\text {Dra }}$ are a useful repackaging of the information in the above scale-shape split objects. Then

$$
K_{\mathrm{K}}:=\left\{\mathrm{F}_{\mathrm{K}}\left[\mathrm{Dra}^{i}, \Pi_{i}^{\text {Dra }}\right]\right\}
$$

See [6] for their relational quadrilateral counterparts and [7] for the relational $N$-a-gon case covered in less detail. 


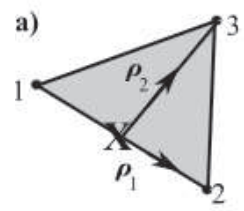

b)

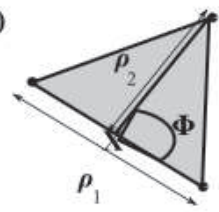

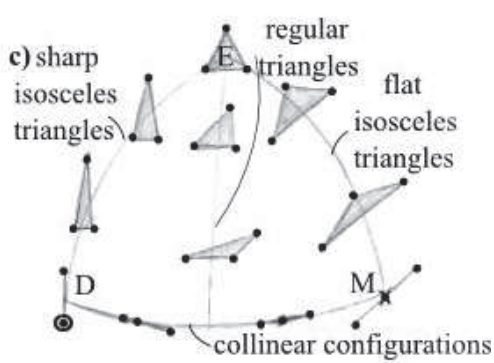

Figure 2. The relational triangle. a) Relative Jacobi vectors. $\mathrm{X}$ is the centre of mass of particles 1 and 2. b) Their magnitudes, base and median labels, and the angle between them. c) The triangleland sphere, with what triangles correspond to which points, is 6 copies of the given $1 / 3$-hemisphere: Kendall's spherical blackboard [7]. The 6 copies correspond to different possible labellings of the triangle. $\mathrm{D}$ is a double collision, $\mathrm{M}$ is a merger and $\mathrm{E}$ is the equilateral triangle configuration. In comparison with Wheeler's well-known depiction of Superspace $(\Sigma)$ [168], both are clearly spaces of spaces, but the relational triangle's clearly has the simpler mathematics that renders it of further use as a model arena.

Example 1. i) The electric field $\underline{E}$ and the magnetic field $\underline{B}$ are Kuchar̆ beables for electromagnetism. ii) The Wilson loops

$$
W_{\gamma}\left[A^{i}\right]=\exp \left(i \oint_{\gamma} A_{i}(y) \mathrm{d} y^{i}\right)
$$

(here $\gamma$ is a path in space) are also Kuchař beables for electromagnetism. Furthermore ii) generalizes to Yang-Mills theory upon introducing tracing over the internal indices. These loop variables form an overcomplete set of such beables (there are Mandelstam identities between them); this point is well-covered in e.g. [67]. As regards the significance of this example, the counterpart of such loops in the Ashtekar variables case are indeed the loops in loop quantum gravity.

Formal strategy 1) One can also act with $\mathfrak{g}$ and then perform an operation involving the whole of $\mathfrak{g}$ (e.g. summing, integrating, averaging, taking an inf, sup or extremum) in order to construct formally $\mathfrak{g}$-invariant expressions that serve as Kuchař beables [10].

Formal strategy 2) In some cases also one knows formally what the $\mathfrak{g}$-invariant expressions are. 'Formal' here refers to not having a concrete basis of these such as the above Hopf-Dragt coordinates for triangleland.

Example 3 or 4. For GR as geometrodynamics the classical Kuchař beables are, formally as per strategy 2), functionals of the 3-geometries and associated momenta,

$$
K_{\mathrm{K}}=\left\{\mathrm{F}_{\mathrm{K}}\left[\mathfrak{G} \text { eom, } \Pi^{\mathfrak{G e o m}}\right]\right\} .
$$

Following strategy 1) instead, one can use entities integrated over all space (but they are not local) or integrated over $\operatorname{Diff}(\Sigma)$ (but the measure of integration in such expressions remains formal).

Example 5. For Ashtekar variables formulations of GR, to commute with $\mathcal{M}_{i}$ in addition to with $\mathcal{G}_{I}$, one needs to consider the diffeomorphism-invariant classes of loops; this coincides with the mathematical definition of knots. Classical Kuchar beables are then, formally in the sense of strategy 2), functionals of knots and associated momenta,

$$
K_{\mathrm{K}}=\left\{\mathrm{F}_{\mathrm{K}}\left[\mathfrak{K}_{\mathrm{Knot}}, \Pi^{\mathfrak{K} \text { not }}\right]\right\} .
$$

Example 6. The more standard (canonically untransformed) bein presentation of GR involves using the configuration space $\operatorname{Bein}(\Sigma)$ in place of $\operatorname{Riem}(\Sigma)$. Then $\operatorname{Diff}(\Sigma)$ and the local 
Lorentz transformations are quotiented out in order to pass to a reformulation of the information contained in $\operatorname{Superspace}(\Sigma)$. Nor is this an empty variant of formalism since inclusion of fermions (e.g. Einstein-Dirac theory) requires reformulation away from metric variables.

Example 7. For supergravity, one cannot just quotient out the linear constraint generated supersymmetric generalization of $\operatorname{Diff}(\Sigma)$ because of of $\mathcal{L} \mathcal{I} \mathcal{N}_{\mathrm{L}}$ not forming a subalgebraic structure of constraints. Thus 'SuperSuperspace $(\Sigma)$ ' - the naïve supersymmetric generalization of Wheeler's Superspace $(\Sigma)$ for GR as geometrodynamics - turns out not to be well-defined. In this case, one has to consider the fully reduced configuration space and the full notion of Dirac beables as per Section 3 .

Example 8. (subcase of geometrodynamics of cosmological relevance). Kuchař beables for perturbatively inhomogeneous cosmology about a homogeneous isotropic $\mathbb{S}^{3}$ minisuperspace with single scalar field matter are exposited in [11] based on the earlier work in [30, 81, 85, 111, $117,143,163,164]$. These are in terms of a countable infinity of mode coefficients for the small perturbations. These constitute an explicit $\mathbb{S}^{3}$ basis much like the Hopf-Dragt coordinates for relational triangle. This demonstrates how some regimes of GR are simpler and are usefully modelled by relational particle mechanics such as this review's relational triangle model.

Example 9. Other models for which Kuchař beables are known include a few midisuperspaces (inhomogeneous but still nontrivially symmetric models that are more amenable to calculations than fully general models). For instance, a) some spatially compact without boundary Gowdy models [157]. These are once again functions of an infinite number of mode coefficients. b) Some open ${ }^{15}$ midisuperspace models with known Kuchař beables are the cylindrical gravitational wave [104] and spherically symmetric gravitational models [108].

\section{Classical Dirac beables}

\subsection{Motivation for Dirac beables}

Perhaps instead the problem of beables is to be resolved by finding Dirac beables. These however may be difficult objects to construct in practise. E.g. explicit construction of Dirac beables is subject to the caveat of requiring explicit solution of a model's dynamics [90, 144], which is in general blocked due to the onset of chaos. Each of the Kuchar beables and partial observables positions can be interpreted as a halfway houses toward construction of Dirac beables. The former is clearly by applying one further partial differential equations restriction to one's set of Kuchar beables: the commutation also with the quadratic constraint. The latter is via methods developed by Dittrich and Thiemann [57, 58, 155].

\subsection{Examples of Dirac beables problems posed}

Example 1. In electromagnetism, Yang-Mills theory and the gauge theories associated with each, Dirac is equivalent to Kuchař for beables, so just take what is said in Section 2 about these theories' beables. This is also the case for temporally-absolute configurationally-relational mechanics.

Example 0. There is just one condition to be solved for each of spatially absolute mechanics and minisuperspace:

$$
\left\{\mathcal{E}, D_{\mathrm{D}}\right\} \approx 0, \quad\left\{\mathcal{H}, D_{\mathrm{D}}\right\} \approx 0 .
$$

\footnotetext{
${ }^{15}$ In Examples 8 and 9, I just give citations to keep this review of manageable length. Aside from here, I also restrict this review to universes that are spatially compact without boundary. Asymptotically-flat models have further notions of asymptotic observables/beables and interior observables/beables. Also far from all open models are asymptotically flat, so the study widens further upon consideration of open models. Likewise we do not have space in this review to consider the notion of observables/beables in holographic theories.
} 
Example 2. Relational particle mechanics have just one more condition to be solved on top of an already-solved set of conditions $(2.1)$, (2.2) with $K_{\mathrm{K}} \longrightarrow D_{\mathrm{D}}$. It is schematically also of the form (3.1).

Example 3 or 4. GR as geometrodynamics and in terms of Ashtekar variables both have just one more condition to be solved on top of a given set of conditions, the $K_{\mathrm{K}} \longrightarrow D_{\mathrm{D}}$ of $(2.3)$ and (2.4) respectively.

Example 7. Having presented a reason why the problem of finding Kuchař beables/observables for supergravity is not well-defined, I now pose the question of finding a full set of classical and then quantum Dirac beables/observables for supergravity. This in fact appears to be a new question, just beyond the frontier in [52] of finishing to construct the classical Dirac brackets algebra for supergravity.

\subsection{Examples of Dirac beables problems solved}

For Example 0, i) See e.g. [19] for a direct construction of classical Dirac beables for minisuperspace.

ii) Halliwell [77] gave a classical-level construct; for a simple $k$ - $d$ particle mechanics model and $\delta^{(k)}$ the $k$ - $d$ delta function, it is of the form

$$
A\left(\mathbf{q}, \mathbf{q}_{0}, \boldsymbol{p}_{0}\right)=\int_{-\infty}^{+\infty} \mathrm{d} t \delta^{(k)}\left(\mathbf{q}-\mathbf{q}^{\mathrm{cl}}(t)\right)
$$

Here $\mathbf{q}^{\mathrm{cl}}(t)$ is a configuration space vector valued classical solution labelled by initial data $\mathbf{q}_{0}, \boldsymbol{p}_{0}$. It is necessary in this construct to treat the whole path rather than just segments of it. This is because elsewise the endpoints of segments contribute right-hand-side terms to $\{\mathcal{H}, A\}$. Whilst these Dirac beables are built out of histories, the final constructs themselves are integrals over all times, by which these are indeed beables as opposed to histories beables (see Section 7.2 for these). This construct extends both to minisuperspace GR [77, 79, 80] and to the triangleland relational particle mechanics [3] subcase of Example 2 formulated in terms of its Kuchar beables (2.6), which provides a solution to Example 2. The latter case involves use of the three Hopf-Dragt coordinates of (2.5) in place of the 3- $d$ case of $\mathbf{q}$, Thus additionally it is an example of building on the halfway house of having constructed a set of Kuchař beables.

For Example 3 or 4, as regards GR beyond minisuperspace, i) see [11] for some Dirac beables for the Halliwell-Hawking model.

ii) Dirac beables are sometimes also explicitly known [157] for some of the Gowdy midisuperspace models.

iv) In outline, Dittrich's [57, 58] general formal power series expansion objects for GR are of the form

$$
D_{\phi}=\sum_{n=0}^{\infty} \frac{1}{n !}\{\mathrm{F}\}^{n}\{\phi, \overline{\mathcal{C}}\}_{(n)} .
$$

Here $\phi$ are dynamical fields, $\mathrm{F}^{\mu}:=X^{\mu}-Y^{\mu}\left(X^{\mu}\right)$ is a gauge-fixing equation for $Y^{\mu}$ spacetime scalar functions, and $\overline{\mathcal{C}}_{\mu}$ are particular linear combinations of the GR constraints [88]. Also $\{,\}_{(n)}$ is an ' $n$ times iterated Poisson bracket', i.e. $n$ Poisson brackets nested inside each other. Each $\overline{\mathcal{C}}_{\mu}$ is contracted with that on one power of $F^{\mu}$. See $[57,58]$ for the conceptually relevant points of how this construct 1 ) exemplifies proceeding to Dirac beables via a partial observables halfway house. 2) That it involves some partial observable acting as a clock variable for the others. See also $[59,60]$ for an outline of this perturbative approach in which an Abelian set of constraints is iteratively produced, alongside the application of this construction to the important case of inhomogeneous cosmological perturbations. This approach has already been recently covered in $[57,58,131,152,155]$, so I detail it here no further. 
The explicit construction problems of Section 3.1 do not affect Halliwell's formal expressions integrated over all time, but do also apply to Dittrich's power-series construct.

\section{Local observables and beables: fashionables and degradeables}

That coordinates are not in general globally defined on closed configuration spaces follows from $\mathbb{S}^{2}$ being e.g. the shape space for 3 particles in 2- $d$. Furthermore, classical beables brackets are partial differential equation conditions. Partial differential equation solutions seldom form a globally-coherent whole (e.g. they seldom admit global well-posedness or explicit global solutions). This is far more serious a mathematical problem than a mere patching together of coordinates.

Fashionables are observables that are local in time and space.

Degradeables, on the other hand are beables that are local in time and space.

These are fitting nomenclature for local versions of these concepts along the lines of the expressions 'fashionable in Italy', 'fashionable in the 1960's', 'degradeable within a year' and 'degradeable outside of the fridge' all making good sense. Additionally, fashion is in the eye of the beholder - observer-tied, whereas degradeability is a mere matter of being rather than of any observing. Note that the above use in detail of this nomenclature is my own [3]. Bojowald et al. $[43,44,89,91]$ had previously introduced the term 'fashionables' without making distinction between observables and beables, i.e. their use of the name 'fashionables' covers both of my uses of 'fashionables' and 'degradeables'. Moreover, [43, 44, 89, 91] also provide computations for these quantities, which serve for either of these interpretations, thus also providing a means of constructing what I term degradeables if one adopts a realist interpretation of QM. [43, 44, 89, 91] furthermore exemplify patching. Patching quite clearly ties well with the partial observables approach, though this notion applies also to the Kuchař and Dirac conceptualizations of observables or beables. See Section 6 for further details of Bojowald et al. work at the quantum level.

As further examples, in fact, examples of Section 2 are in general but Kuchař degradeables rather than globally well defined Kuchař beables.

Also, Dittrich's power series construct depends on the 'clock variable' conjugate to the constraint being well-defined, which is in general only local.

Finally, Halliwell's integration over all time construct has the global problem that one's choice of time function does not generally hold throughout space or for all values of that time. There is an issue of operational useabilty for entities that require evaluation over all of history. Non-globality of many emergent and hidden times clashes with how the version of Halliwell's construct based on integrating over finite time intervals fails to commute with $\mathcal{H}$.

\section{Diffeomorphism-specific issues with classical beables}

Passing from ordinary gauge theory to GR substantial increases conceptual and technical complexity. I begin by recollecting two early no-go results about Dirac beables.

\subsection{Kuchař's and Torre's no-go theorems}

Kuchař's no-go theorem [105]. Nonlocal objects of the form

$$
\int_{\Sigma} \mathrm{d}^{3} x \mathrm{~K}_{i j}\left(x^{k} ; h_{l m}\right] p^{i j}(\underline{x})
$$

are not Dirac beables (for $\mathrm{K}_{i j}$ some general spatial tensor-valued mixed function-functional). This result makes use that metric concomitants are in general built out of covariant derivatives 
of the Riemann tensor. It then proceeds by proving inductively on the number of covariant derivatives that $\mathrm{K}_{i j}$ cannot contain concomitants with that number of covariant derivatives, by use of algebraic and integrability arguments.

Torre's no-go theorem [156] (see also [14, 49, 158]). Local functionals

$$
\mathrm{T}\left(x^{i} ; h_{k l}, p^{n m}\right]
$$

are not Dirac beables either. This uses that local observables correspond to local 'hidden symmetry' but that the latter's cohomological classification then leaves no viable options.

\subsection{Interpretations of diffeomorphisms}

For $\mathfrak{s}$ some differential manifold, I make standard use of $\operatorname{Diff}(\mathfrak{s})$ for the diffeomorphisms (usually actively interpreted). $\operatorname{Diff}(\mathfrak{m})$ and $\operatorname{Diff}(\Sigma)$ are then cases of particular relevance.

$$
\operatorname{Diff}(\mathfrak{m})=\left\{\epsilon\left(X^{\mu}\right)\right\}
$$

corresponding to coordinate transformations ${ }^{16} X^{\mu} \rightarrow \widetilde{X}^{\mu}=f^{\mu}\left(X^{\nu}\right)$. However GR is invariant under a larger group [39]: the diffeomorphism-induced gauge group,

$$
\operatorname{Digg}(\mathfrak{m}):=\left\{\epsilon\left(X^{\mu} ; \phi^{\Gamma}\left(X^{\mu}\right)\right]\right\} .
$$

Here $\phi^{\Gamma}\left(X^{\mu}\right)$ denote the fields in one's theory (metric $g_{\mu \nu}\left(X^{\mu}\right)$ and matter fields). Digg( $\left.\mathfrak{m}\right)$ might also be denoted BK(m) after Bergmann and Komar [39], though they themselves referred to it as the ' $Q$-group', and Pons, Salisbury and Sundermeyer prefer to use the Bergmann-Komar name for the final group in this subsection. Incidentally, the existence of this larger invariance does not by itself dictate that it is the gauge group. One can argue rather for freedom to choose different suitably compatible groups to be physically irrelevant, and then consider the outcome of each theory [13]. Inconsistencies and observational unviability then serve to kill off choices not realized in nature. Adopting $\operatorname{Digg}(\mathfrak{m})$ invariance feeds into one type of resolution of the frozen formalism problem (see Appendix A), so there are theoretical reasons for considering this group.

Next,

$$
\operatorname{Data}(\mathfrak{m}):=\left\{\epsilon\left(X^{\mu} ; \phi_{\mathrm{CD}}^{\Gamma}\left(X^{\mu}\right)\right]\right\} .
$$

Here 'CD' denotes 'depends on the fields only via the Cauchy data on a spatial hypersurface $\Sigma$. This notion was adopted by Dirac, and so Bergmann-Komar called it 'D-group'. The associated invariance concerns transformations that are unchanged under 4- $d$ coordinate transformations that reduce to it on initial $\Sigma$ on which the canonical Cauchy data are defined. E.g. any spatial 3 -vector, $h_{a b}$ or $p^{a b}$ are Data-invariant. Dirac adopted this since his application of Poisson brackets is appropriate to $\operatorname{Data}(\mathfrak{m})$ but not to $\operatorname{Diff}(\mathfrak{m})$ or $\operatorname{Digg}(\mathfrak{m})$.

I denote the final group of particular interest by $\operatorname{PDigg}(\mathfrak{m})$ : the projected version of $\operatorname{Digg}(\mathfrak{m})$. Pons, Salisbury and Sundermeyer [131] refer to this as the Bergmann-Komar group, since it is also given in [39]. However, it is surely more natural in talking about the two new groups in [39] to name this one by its distinctive feature of being a projection. This is as opposed to trying to find a less clear distinctive feature that refers to the larger group. I note moreover

\footnotetext{
${ }^{16}$ Infinitesimal transformations $X^{\mu} \rightarrow \widetilde{X}^{\mu}$ can be written as $X^{\mu}-\widetilde{X}^{\mu}=\epsilon^{\mu}$. Viewed as solutions in terms of phase space variables, the right hand side functions here are so-called descriptors (a fairly standard gaugetheoretic notion, see e.g. [15, 39]). For GR, descriptors are arbitrary functions of $X^{\mu}, h_{i j}$ but not of $N$ or $N^{i}$. Section 3.3's $\mathrm{F}^{\mu}$, the below $\xi^{\mu}$ and Section 5.5's Weyl scalars can each be viewed as particular uses of descriptors.
} 
that Bergmann and Komar themselves did not themselves know about this group's geometrical interpretation in terms of projection. This was, rather, later elucidated by Pons, Salisbury and Shepley [128]. Before considering this interpretation, it is worth pointing out the form of the group:

$$
\operatorname{PDigg}(\mathfrak{m})=\left\{\epsilon\left(X^{\mu} ; \phi^{\Gamma}\left(X^{\mu}\right)\right] \epsilon \operatorname{Digg}(\mathfrak{m}) \mid \epsilon=n^{\mu}\left(X^{\nu}\right) \xi^{0}-\delta_{a}^{\mu} \xi^{a}\right\} .
$$

Here, the $\xi^{\mu}$ are descriptors (see footnote 16) of the particular form $\xi^{\mu}\left(X^{\mu} ; \phi^{\mathrm{P}}\left(X^{\mu}\right)\right] . \phi^{\mathrm{P}}$ denotes a set of metric and matter fields that now specifically exclude the GR lapse and shift. Finally, $\mathcal{H}_{\mu}$ denotes the 4 -vector of constraints $\left[\mathcal{H}, \mathcal{M}_{i}\right]$. Then the projecting in question is from configuration-velocity space to phase space, i.e. associated with a Legendre map. It effectively means that the induced gauge group for $Q^{\mathrm{A}}, P_{\mathrm{A}}$ is smaller than that for $Q^{\mathrm{A}}, \dot{Q}_{\mathrm{A}}$. Thus PDigg's 'P' can be taken to stand for 'phase' as well as for 'projection'. In effect, $\operatorname{Digg}(\mathfrak{m})$ itself cannot be completely realized in phase space (and $\operatorname{Diff}(\mathfrak{m})$ is not meaningful in this sense either); this is what motivates adopting $\operatorname{PDigg}(\mathfrak{m})$ instead. The corresponding active canonical phase space transformation is (see Fig. 1 for the meaning of $P_{\mu}$ )

$$
G_{\xi}=P_{\mu} \dot{\xi}^{\mu}+\left\{\mathcal{H}_{\mu}+N^{\rho} f_{\mu \rho}^{\nu} P_{\nu}\right\} \xi^{\mu}
$$

Also here, $f_{\mu \rho}^{\nu}\left(h_{i j}\right)$ are the Dirac algebroid's structure functions wrapped up in the spacetime tensor form corresponding to expressing the constraints as a spacetime vector $\mathcal{H}_{\mu}$.

Bergmann and Komar [39] also posited a number of relations between the groups involved. This led them to conclude that Dirac and Bergmann beables turn out to coincide, but they provide no proofs for the underlying relations and this claim has since been contested $[114,169]$.

\subsection{The difference between a Hamiltonian and a gauge generator}

The next two subsections are based on the 'scholium' presented in dialogue form [130, pp. 21-22], and in Section 3.3 of [131].

The 'evolution' generator $\delta t\left\{N^{\mu} H_{\mu}+\dot{N}^{\mu} P_{\mu}\right\}$ does serve to replace solutions at time $t$ by the original solutions evaluated at $t-\mathrm{d} t$. However, this is merely its action on one particular member of each equivalence class of solutions. I.e. the particular member for which the lapse and shift form the chosen explicit function $N^{\mu}$ as per Fig. 1. Its action on all other members of these equivalence classes generates variations different from global time translations.

In more detail, points $p \in S=\{$ the space of the dynamical fields $\phi\}$ are specific spacetimes plus matter fields when relevant: solutions of the equations of motion as described in a particular coordinatization. Also use D to denote the data for the dynamical fields on some spatial hypersurface that is labelled by 'initial time' $t_{0}$. Given a specific selection of the arbitrary functions of the dynamical variables $\lambda^{\mu}$, the corresponding Dirac Hamiltonian is $\mathrm{H}(t)=N^{\mu} \mathcal{H}_{\mu}+\lambda^{\mu} P_{\mu}$. This dictates - via the Poisson brackets - the time evolution in $p$. In particular, for an infinitesimal $\mathrm{d} t$, this Hamiltonian gives what the field data $\mathrm{D}^{\prime}$ are on the subsequent spatial hypersurface labelled by $t_{0}+\delta t$. If we carry out this procedure for all times $t$, it of course results in a 'null operation': we have remained exactly at the same point $p \in S$. This simply reflects that the dynamics as described by a given observer takes place within a given spacetime in a given coordinatization.

Next consider the gauge generator that, after suitable choice of the descriptors, happens to coincide in its mathematical form with the Dirac Hamiltonian at time $t_{0}$. By this coincidence, its action likewise transforms the field data $\mathrm{D}$ into $\mathrm{D}^{\prime}$. However, these data $\mathrm{D}^{\prime}$ are now to be interpreted at time $t_{0}$, since the notion of gauge transformations in question are equal-time actions. What has occurred is that we have moved from $p$ to another, albeit gauge-equivalent, spacetime $p^{\prime}$. (I.e. it is mathematically another point in $S$, but it corresponds to the same physics.) Then suppose we undertake the same procedure for any time $t$ whilst continuing to 
assume that the descriptors at time $t$ match up with the lapse and shift at $t$. Then we end up having mapped the whole spacetime $p$ to $p^{\prime}$. Notice that the field configurations in $p$ and $p^{\prime}$ differ solely as regards their time labels. Thus a passive diffeomorphism $t \rightarrow t-\delta t$ renders both descriptions mathematically identical. This demonstrates that the gauge generator's capacity to mimic the Hamiltonian is conceptually unrelated to there being real physical evolution in a given spacetime $p$. Thus dynamical evolution in $p$ is not the same thing as gauge action on $p$.

\subsection{The 'nothing happens' fallacy}

'(1.13) means that nothing happens' is a common type of frozen argument (Appendix A discusses others). However, the inference that 'nothing happens' is a fallacy on the following grounds [130].

On the one hand, following from the preceding subsection, we have an 'evolved configuration' $\mathrm{D}^{\prime}$ lying to the future of of an 'initial configuration' $\mathrm{D}$. On the other hand, $\mathrm{D}$ and $\mathrm{D}^{\prime}$ are related by a gauge transformation. Since 'gauge transformations do not change the physics', we deduce that 'the physics' in $\mathrm{D}$ and $\mathrm{D}^{\prime}$ are the same. So the future configuration is gaugeequivalent to the initial configuration and therefore 'nothing happens'. The fallacy comes from each of the two hands using a single common language for two sets of things that are in fact conceptually different in each case. Recollect from Section 1.3 that there are two notions of gauge transformation: Dirac's and Bergmann's. There are furthermore also two corresponding notions of 'the physics'. The second hand involves mapping solutions of the equations of motion to other such solutions, and so requires the 'entire field configurations' of a 'whole-path', 'wholehistory' or 'whole-spacetime' physics perspective, and thus involves Bergmann's notion of gauge. In contrast, the first hand involves 'configurations at a given time $t_{0}$ ' ( $\mathrm{D}$ and $\left.\mathrm{D}^{\prime}\right)$, i.e. a 'timesliced' physics' dynamical perspective, and thus involves Dirac's notion of gauge. Thus the two hands in fact use both distinct notions of 'gauge' and corresponding distinct notions of 'the physics'. Since the 'nothing happens' argument does not take these differences into account, it is rendered fallacious. See [98, 127, 129, 155] for further support of this point. Finally, this resolution of the 'nothing happens paradox' corresponds to the distinction between timedependent beables $D_{\mathrm{D}}(t)$ for $t$ an intrinsic coordinate scalar that constitutes a gauge fixing, as opposed to just a constant $D_{\mathrm{D}}$ as occurred in the opening paragraph (see Section 3.4 of [131] for more on this point). Clearly the former are not 'constants of the motion'!

\subsection{Using Weyl scalars as observables/beables}

The Weyl scalars are spacetime scalars; they are built from the Weyl tensor irreducible part of the spacetime Riemann tensor. See [69] for their detailed forms (not needed for this review's discussion). The Weyl scalars are of value as per below, as well as being foundational for the Newman-Penrose formulation of GR [122, 123].

The Weyl scalars can additionally be considered as a concrete proposal [39, 127] for observables/beables in the sense of Bergmann. Indeed Bergmann and Komar ${ }^{17}$ converted the spatial components of the spacetime Riemann tensor and contractions with the spatial hypersurface normal $n^{\mu}$ to be purely in terms of canonical variables (meaning in this case $h_{a b}, p^{a b}$ and not $N$ or $N^{i}$ ). Thus the Weyl scalars can be written in terms of the canonical variables, as befits many of the expectations about observables/beables. In this application, they are to be interpreted as intrinsic coordinates, and also as 'making use of a set of scalars as a gauge fixing'. Finally note that these scalars can in principle be observed locally and in a convenient manner, e.g. by use of a Szekeres gravitational compass [149].

\footnotetext{
${ }^{17}$ Note that while this subsection involves the same authors as the previous four subsections it concerns a largely disjoint idea.
} 


\subsection{Comments}

Revisiting Torre's no-go theorem. [127] posits that Torre proves nonexistence of constantin-time observables $=$ constants of the motion, built as spatial integrals. This is as opposed to dealing with Bergmann observables such as the Weyl scalars. On the other hand, Dittrich and Thiemann's approach [57, 58, 155] gets round the Torre no-go by [130, 131] involving series of Cauchy data derivatives that are in principle up to infinite order. Finally, Halliwell's classical construct avoids Torre's by not being local in space or time and it avoids Kuchařs by not being of form (5.1).

Connection with partial observables approaches. [127] is supportive as is [130] as per above, and [129]. [130] does limit support in the sense of insisting partial observables be spacetime scalars (a standard tenet of internal time approaches). However [127] argues for Weyl scalars exemplifying partial observables (which of course do in this case comply with being spacetime scalars). Thus Dittrich and Thiemann's works on observables/beables has wider conceptual support/motivation than is often acknowledged. I.e. these avoid the Kuchař and Torre no-go results and are aligned with the works of Pons, Salisbury, Shepley and Sundermeyer and preceding works by Bergmann and Komar.

Reparametrization-invariant model counterpart. Some of this section's issues already have nontrivial counterparts for RI theories. In place of $\operatorname{Diff}(\mathfrak{m})$, one has

$$
\text { (reparametrizations) } \quad \mathrm{R}=\{\epsilon(t)\},
$$

and in place of in place of $\operatorname{Digg}(\mathfrak{m})$, one has

$$
\operatorname{Rigg}=\left\{\epsilon\left(t ; q^{i}(t)\right]\right\}
$$

(reparametrization-induced gauge group). Finally, in place of $\operatorname{PDigg}(\mathfrak{m})$, one has

$$
\text { PRigg }=\left\{\epsilon\left(t ; q^{i}(t)\right] \in \operatorname{Rigg} \mid \epsilon=\xi\left(t ; q^{i}(t)\right] / N\right\} .
$$

(That is obtained by applying the formula for $n^{\mu}$ in Fig. 1 to (5.2) and truncating to 1-d.) How complete these reparametrization invariant counterparts are as a model of diffeomorphisminduced groups remains to be worked out in detail.

Also note that Lee-Wald's tie between $\operatorname{Digg}(\mathfrak{m})$ nontriviality and GR algebroid fails to work for Rigg, limiting the extent to which Rigg functions as a toy model for $\operatorname{Digg}(\mathfrak{m})$.

Pitts' work. I finally mention to keep an eye on Pitts' concurrently produced work that follows in part from the work from Bergmann through to Pons et al., starting with [125].

\section{Beables at the quantum level}

The simplest notion of QM observables concerns self-adjoint operators which therefore have real eigenvalues and to that extent are realistic. This is prior to gauge-theoretic considerations, which are a further restriction on such operators.

Strategy 1. One has classical beables beforehand, one can attempt to promote them to quantum ones. This might occur at the level of kinematical quantization [92] or be viewed as a process in addition to that. In either case, one finds that one needs to select a classical subalgebra of objects to promote to quantum operators. Perfectly good classical beables can fail to be quantum beables. This parallels how perfectly good classical symmetries fail to be quantum symmetries due to anomalies arising, in the sense that both are bracket obstructions upon passing from classical to quantum brackets. Thus one might term the above phenomenon a 'beables anomaly'. Schematically, for whichever appropriate pairing of classical and quantum types of bracket,

$$
\left\{B, \mathcal{C}_{\mathrm{C}}\right\}^{\prime}={ }^{\prime} 0 \quad \Rightarrow \quad\left[\widehat{B}, \widehat{\mathcal{C}}_{\mathrm{C}}\right] \Psi=0 .
$$


Whether this occurs is moreover dependent twice over on operator-ordering ambiguities (in the beable operators and in the constraint operators). The outcome of the preceding furthermore heavily depends on choice of operator ordering assumed. Additionally, trying to promote known classical beables to quantum ones also falls afoul of the multiple choice problem (see Appendix A).

Strategy 2. One might also start afresh in the quest to find observables/beables at the quantum level. This makes particular sense upon realizing that in general the classical and quantum brackets correspond to different algebras due to global effects entering at the quantum level [92]. In general, the entities that commuted with the classical constraints with respect to one brackets structure should not be expected to result in quantum operators that commute with the quantum constraints with respect to an algebraically-distinct brackets structure!

An issue affecting both strategies in the case of loop quantum gravity is that one has yet to attain a satisfactory form for the quantum Hamiltonian constraint, which would then be required as an entity that enters the definition of Dirac beables. Another issue affecting both strategies for any sufficiently general theory is as follows. Whereas strategy 1 directly follows on from what is in general a practical impossibility - general classical solution of equations as complicated as the Einstein field equations of GR - it is hard to believe that strategy 2's starting afresh at the quantum level would simplify this problem. Indeed, Dirac [56] commented that QM seldom simplifies situations that are already complicated at the classical level.

The quantum problem of beables is then that, whichever of the above strategies one employs, it is hard to come up with a sufficient set of these for quantum-gravitational physics.

\subsection{Resolution of Heisenberg picture form of frozen formalism problem}

Adopting Section 5.4's interpretation of the 'nothing happens paradox' entails resolution of the quantum frozen formalism problem in its Heisenberg picture form. If this problem is resolved in the Heisenberg picture, it would often also be argued that it is resolved in the Schrödinger picture due to these two pictures standardly being unitarily equivalent. On the other hand, Rovelli would appear to differ in this regard [137], by giving the Heisenberg picture a privileged status in quantum gravity.

The existence of a Heisenberg resolution furthermore demotivates timeless approaches such as the naïve Schrödinger interpretation [86, 159], conditional probabilities interpretation [121], Page's approach [119, 120], Gambini-Porto-Pullin's [65, 66], Barbour's [24, 27] and other forms of fully timeless records theory [2,9] (see Appendix A for this terminology).

On the other hand, I argued that histories theory (also see Appendix A) is distinct in this regard, as are 'time from change' approaches [8, 26, 137]. There are possibly additionally positions intermediate between the previous two sentences'. E.g. the well-known path integral approaches and 'records within histories' approaches [2, 3, 9, 70, 77, 78, 79, 80].

Finally note that such a frozen formalism problem resolution does not amount to a resolution of the quantum problem of beables itself.

\subsection{Examples of quantum beables}

Quantum Dirac beables obey (1.14), where all second-class constraints have been priorly dealt with at the classical level by Dirac brackets or extension to effective phase space. On the other hand, quantum Kuchař beables obey (1.15), and are applicable in cases for which the $\overline{\mathcal{L I N}}_{\mathrm{L}}$ form a closed algebraic structure under quantum commutators.

Examples 0 and 2. In the absense of any linear constraints, Kuchař beables remain trivial at the quantum level. [19] also gives some explicit examples of minisuperspace quantum Dirac beables. Explicit quantum Kuchař beables for the relational triangle follow from adopting the 
Hopf-Dragt coordinates, their conjugate momenta and an $\mathrm{SO}(3)$ 's worth of shape momenta as the kinematical quantization for that model [3].

On the other hand, some general features of indirect constructs are as follows.

Universal Example 1. Any operator $\widehat{O}$ can be inserted into the construction

$$
\widehat{O}_{\mathrm{D}}:=\int \mathrm{d} t \exp \left(i H_{0} t\right) \widehat{O} \exp \left(-i H_{0} t\right)
$$

for a suitable notion of time (e.g. label time $\lambda$ in minisuperspace or Newtonian time in mechanics), which again needs to run over all values of time rather than just some interval. Formal field-theoretic generalizations of this construct are also straightforward. DeWitt's [54] early treatment of construction (6.1) further specialized to the semiclassical case. Marolf [115] then treated such objects in the case of QM at the perturbative level.

Example 3. Another means of attaining observables/beables in the case of GR, exposited by Giddings, Marolf and Hartle, involves integrating $\widehat{O}$ over all of spacetime [71]. These authors consider attaining locality by smearing with delta functions.

Universal Example 2. Halliwell's semiclassical Dirac beables construction for theory with no linear constraints consists of the class functional $^{18}$

$$
\widehat{C}_{\mathrm{R}}\left[\mathbf{q}_{\mathrm{f}}, \mathbf{q}_{0}\right]:=\theta\left(\int_{-\infty}^{\infty} \mathrm{d} t f_{\mathrm{R}}\left(\mathbf{q}_{\mathrm{f}}(t)\right)-\varepsilon\right) P\left(\mathbf{q}_{\mathrm{f}}, \mathbf{q}_{0}\right) \exp \left(i S\left(\mathbf{q}_{\mathrm{f}}, \mathbf{q}_{0}\right)\right) .
$$

Once again, the specific example is mechanics but has also been considered for minisuperspace [77] and triangleland relational particle mechanics [3]. For the first two cases, it is a $S$ matrix construct since there are no linear constraints; in the last case it is a nontrivial Dirac beables construct since this case has linear constraints to overcome too. Note that this is an example of 'starting afresh' with a new structure rather than of trying to promote Halliwell's distinct classical construct to the quantum level. The class functional can then be used to re-express the decoherence functional between pairs of histories $\eta, \eta^{\prime 19}$

$$
\begin{aligned}
\mathcal{D e c}\left[\eta, \eta^{\prime}\right] & =\int_{\eta} \mathbb{D} \mathbf{q} \int_{\eta^{\prime}} \mathbb{D} \mathbf{q}^{\prime} \exp \left(i\left\{S[\mathbf{q}(t)]-S\left[\mathbf{q}^{\prime}(t)\right]\right\} \rho\left(\mathbf{q}_{0}, \mathbf{q}_{0}^{\prime}\right)\right. \\
& =\iiint \mathbb{D} \mathbf{q}_{\mathbf{f}} \mathbb{D} \mathbf{q}_{0} \mathbb{D} \mathbf{q}_{\mathrm{f}}^{\prime} \widehat{\mathcal{C}}_{\eta}\left[\mathbf{q}_{\mathbf{f}}, \mathbf{q}_{0}\right] \widehat{\mathcal{C}}_{\eta^{\prime}}\left[\mathbf{q}_{\mathrm{f}}^{\prime}, \mathbf{q}_{0}^{\prime}\right] \Psi\left(\mathbf{q}_{0}\right) \Psi\left(\mathbf{q}_{0}^{\prime}\right) .
\end{aligned}
$$

Note that the semiclassical Halliwell and quantum Marolf constructs indeed provide examples of beables rather than of histories beables (a notion along the lines of Section 7.2). In each case, this is due to the history content being integrated out by the integration over all $t$. Moreover, by involving a $t$-integral, Halliwell's semiclassical object is not local in time, which, as for the classical counterpart, would however be a desirable property in a beable that can be used in practise. As in the classical counterpart, the above object manages to commute with $\widehat{\mathcal{H}}$ only by having its integrals run over all time, which is often incompatible in practise with the global nonexistence of e.g. emergent and hidden timefunctions (Appendix A). On the other hand, Anastopoulos' distinct histories-based construct [1] does not have to involve the whole real line. The end-product of this is, however, a histories beable, whereas Halliwell's construct returns in the end just a beable.

A further concern here is whether difficulties ensue if one subsequently forms functionals out of the Kuchar beables (specifically, class functionals). That provides commutation with $\mathcal{Q U A \mathcal { D }}$, but now a functional of beables is not necessarily an beable compatible with one's

\footnotetext{
${ }^{18}$ Here, $\mathbf{q}^{\text {cl }}(t)$ is the classical trajectory, $\mathbf{q}_{0}, \boldsymbol{p}_{0}$ is initial data, $\theta$ is the step function, $f_{\mathrm{R}}$ is the characteristic function of region $\mathrm{R}, \varepsilon$ is a small real number, $S\left(\mathbf{q}_{\mathbf{f}}, \mathbf{q}_{0}\right)$ is the classical action between $\mathbf{q}_{\mathrm{f}}$ and $\mathbf{q}_{0}$. See [82] for the detailed form of the prefactor function $P$.

${ }^{19}$ Here $\mathbb{D} \mathbf{q}$ is a measure and is a $\rho$ a density matrix.
} 
previously determined beables. Thus the multiple choice problem (Appendix A) is looming as a reason for breakdown of the Halliwell procedure being applied to produce nontrivially-Kuchař Dirac observables. And yet, $\mathcal{L} \mathcal{I} \mathcal{N}_{\mathrm{L}}$ is absent by this stage. Thus it is not $\left[\widehat{\mathcal{L I N}}_{\mathrm{L}}, \widehat{K}_{\mathrm{K}}\right] \Psi=0$ failing to imply that $\left.\left[\widehat{\mathcal{L I N}}_{\mathrm{L}}, \widehat{\mathcal{F}} \overline{\left(\underline{x} ; K_{\mathrm{K}}\right.}\right]\right] \Psi=0$ also holds. Rather it is an issue of whether the class functionals furnish a compatible set among themselves if they are to now be regarded as beables.

Note also that quantization complicates status of beables via the subalgebra selection criterion (and its own associated multiple choice problem). Are the quantum Dirac beables well-defined as functionals of the quantum Kuchař beables?

Additionally, note that Halliwell's 2009 version of class functionals [79] is more complicated than the above treatment but succeeds in additionally avoiding the additional manifestation of frozenness that is the 'watched kettles never boil' quantum zeno problem.

Does using Halliwell's more intricate histories-theoretic machinery have any advantages over DeWitt's and Marolf's constructs? The answer is yes at the level of handling whole-universe issues and problem of time approaches, but whether there is practical equivalence between each approach's type of objects remains uninvestigated.

As a final example, consider Bojowald et al. [43, 44, 89, 91] fashionables approach at the semiclassical level. Here, use is made of a moments expansion to bypass the inner product problem. The fashionables are real-valued, but solving the constraints gives that this approach's notion of time goes complex around the semiclassical regime's turning points. Moreover, in this approach a time variable's imaginary part becoming significant is a diagnostic for that time variable ceasing to be a 'good clock', due to the onset of non-unitarities in the evolution brought about by the global problem of time (Appendix ??).

\section{$7 \quad$ Various further brackets and corresponding notions of beables}

\subsection{Multisymplectic approach}

In this approach (see e.g. [87]), the $\partial / \partial t$ in the standard definition of momentum (1.2) is extended to a $\partial / \partial x^{\mathrm{X}}$ for $\mathbf{X}$ taking $m>1$ values. Then the conventional Hamiltonian curve of the standard $\partial / \partial t$ case, associated with the one tangent vector, is replaced by a multivector notion corresponding to $m$ tangent vectors. The standard case's Poisson bracket's corresponding symplectic form is now replaced by a multisymplectic form. The notion of bracket associated with this is the Schouten-Nijenhuis bracket: a graded Lie bracket on the multivector fields.

The corresponding notion of observables/beables has been considered by Hélein and Kouneiher [87] (for now in a classical, non-whole universe setting in which the observables/beables distinction is not significant).

I finally comment that the multisymplectic approach may have difficulties via putting time and space on even more of an equivalent footing than is usual. Whilst this is in part motivated by a desire to reformulate or replace canonical formulations with manifestly covariant ones, one also has to bear in mind that time and space are conceptually different entities.

\subsection{Histories brackets and histories beables}

Histories theory is motivated as per Appendix A and as regards providing a more natural interpretation for quantum cosmology and a format within which decoherence applies. All of these issues additionally fit in well with realist interpretations of QM and of quantum cosmology. Moreover some versions of histories theory $[97,103,140,141]$ can be viewed as a distinct approach to covariantizing the canonical formulation. In these versions, configurations $Q^{\mathrm{A}}$ have been replaced by whole paths of configurations - classical histories $Q^{\mathrm{A}}(\lambda)$ - in the role of basic canonical entities. Here $\lambda$ is a label time, though it can be replaced with other notions of time 
(Appendix A) without issue. The latter are now associated with histories momenta $P_{\mathrm{A}}(\lambda)$, with histories brackets $\{,\}_{\boldsymbol{H}}$ holding between the two. Thus the entirety of the phase space structure has been replaced by a histories phase space structure. This is an intriguing possibility, though it is not yet clear whether the study of dynamics based on configurations contains any important ingredients that are lost with this paradigm shift to histories being the basic canonical entities. In this approach, additionally, the standard notion of constraints is replaced by that of histories constraints, $\mathcal{C}_{\mathrm{C}}^{\lambda}=\mathcal{C}(\lambda)_{\mathrm{C}}$. There are then obvious generalizations of the notions of first- and second-class constraints, Dirac brackets, extended phase space, effective constraints and constraint algebra, all now in terms of the histories bracket.

Histories beables $[1,103]$ are then histories quantities $B_{\mathrm{B}}^{\lambda}=B(\lambda)_{\mathrm{B}}$ that histories-brackets commute $[97,140]$ with the histories constraints. In particular, in a theory with only first-class constraints, there is a notion of Dirac histories beables that histories-brackets commute with all of these histories brackets constraints,

$$
\left\{D_{\mathrm{H}}^{\lambda}, \mathcal{C}_{\mathrm{C}}^{\lambda}\right\}_{\boldsymbol{H}} \approx 0
$$

In cases in which second-class histories constraints are initially present, this requires using the histories version of the Dirac bracket or reformulation in terms of histories effective constraints. Moreover, some classical theories contain the linear histories constraints $\mathcal{L I N}_{\mathrm{L}}^{\lambda}$ as a subalgebraic structure, alongside a quadratic histories constraint $\mathcal{Q U} \mathcal{A D}^{\lambda}$. In these cases, a notion of histories Kuchař beables is also available:

$$
\left\{K_{\mathrm{H}}^{\lambda}, \mathcal{L} \mathcal{I} \mathcal{N}_{\mathrm{L}}^{\lambda}\right\}_{\boldsymbol{H}} \approx 0
$$

Note 1. Despite both being based on classical paths, the Bergmann and histories notions of beables are technically and conceptually distinct extensions of which gauge groups to attribute to one's physical theory. This is clear from the shift in basic canonical entities in the latter, with the ensuing introduction of a histories brackets structure absent in Bergmann's work and with a number of subsequent parallels to Dirac's notion of beables. Nonetheless, Savvidou [141] showed that histories phase space can also be considered to carry representations of $\operatorname{Digg}(\mathfrak{m})$.

Note 2. The above is a classical precursor for the quantum histories projection operator (HPO) approach [97, 140]. At the quantum level, histories are paths that are furthermore decorated by projection operators. In the HPO approach itself, time is continuous and there is a corresponding continuum of projection operators. Quantum histories commutators $[,]_{\boldsymbol{H}}$ now replace classical histories brackets, and the final set of first-class histories constraints are promoted to quantum operators $\widehat{\mathcal{C}_{\mathrm{F}}^{\lambda}}$. Quantum histories Dirac beables are then further quantum histories operators $\widehat{D}_{\lambda}^{\mathrm{B}}$ such that

$$
\left[\widehat{D}_{\mathrm{H}}^{\lambda}, \widehat{\mathcal{C}}_{\mathrm{F}}^{\lambda}\right]_{\boldsymbol{H}} \Psi=0
$$

On the other hand, for theories with a subalgebraic structure of quantum linear histories constraints $\widehat{\mathcal{L I N}}_{\mathrm{L}}^{\lambda}$, quantum histories Kuchař beables are further quantum histories operators $\widehat{K}_{\mathrm{B}}^{\lambda}$ such that

$$
\left[\widehat{K}_{\mathrm{H}}^{\lambda}, \widehat{\mathcal{L I N}}_{\mathrm{L}}^{\lambda}\right]_{H} \Psi=0
$$

\subsection{Notions of brackets with more slots}

Suppose instead that the pair $Q^{\mathrm{A}}, P_{\mathrm{A}}$ of conventional canonical theory are replaced with $p \geq 3$ different types of entity. Such a theory then possesses $p-1$ scalar entities in place of the standard Hamiltonian. Such a theory is additionally equipped with a generalization of the usual 2-slot 
notion of brackets to a bracket with $p$ slots. Even 3-slot theories are relatively novel in concrete physical examples, so I venture no further.

For theories with brackets with three slots, $|[,]$,$| , it is these that are to be used to determine$ which constraints are first- and second-class, and then also to form the constraint algebra. Then additionally the beables notion forming zero brackets with an entity already in the theory (hitherto the constraints) begins to take multiple forms. Which of

$$
\begin{aligned}
& \left|\left[\mathcal{C}_{\mathrm{C}}, \mathcal{C}_{\mathrm{C}^{\prime}}, B_{\mathrm{B}}\right]\right| \approx 0, \\
& \left|\left[\mathcal{C}_{\mathrm{C}}, B_{\mathrm{B}}, B_{\mathrm{B}^{\prime}}\right]\right| \approx 0
\end{aligned}
$$

(or both) do we use? I point out that it is notion (7.1) that better parallels the standard notion of conserved quantity $C$, which generalizes here to $\mathrm{d} C / \mathrm{d} t=|[C, \mathrm{H}, \mathrm{G}]| \approx 0$ on account of there being two Hamiltonians, $\mathrm{H}$ and $\mathrm{G}$. Perhaps also there are further meaningful notions of bibeables. I.e. entities $B i_{\mathrm{v}}$ such that

$$
\left|\left[\mathcal{C}_{\mathrm{C}}, B_{\mathrm{B}}, B i_{\mathrm{V}}\right]\right| \approx 0
$$

for whichever of the preceding notions of beables. This notion is an analogous concept and nomenclature to how a curve in $3-d$ has a binormal in addition to its normal and its tangent familiar from curves in 2- $d$. The above concepts about beables for 3 -slot brackets indeed suitably extend to $p$-slot brackets. This subsection covers objects of which the next two subsections provide important specific examples from quantum gravity programs.

\subsection{Associator and Nambu brackets, e.g. for M2-branes}

The associator bracket is denoted by $\langle,$,$\rangle and has the algebraic form$

$$
\langle A, B, C\rangle=A \cdot\{B \cdot C\}-\{A \cdot B\} \cdot C
$$

for corresponding notion of plain product operation $\cdot$. I.e. just as the commutator quantifies non-commutativity $([A, B]=A \cdot B-B \cdot A)$, the associator quantifies non-associativity. The algebra thus formed is indeed nonassociative rather than just noncommutative.

Next, the Nambu bracket $[118,151]$ is the completely antisymmetrized associator:

$$
[,,]=\langle,,\rangle+\text { perms }
$$

As an important application, the Bagger-Lambert-Gustavsson action [20, 21, 22, 74] is built out of this bracket. This (Example 10) is an action for multiple $N=8$ M2-branes $^{20}$; this is a leading candidate for understanding the microphysics of M-Theory.

As a simpler example, the octonians are a toy model of the associator bracket. Here $[u, v, w]=$ $\frac{1}{3}\{\{u \times v\} \times w+$ cycles $\}$ renders this bracket in terms of standard mathematics (vector products). Dimension $>3$ is required in order for this not to vanish. Were it to vanish, the condition for it doing so is that the Jacobi identity of the subsequently in general noncommutative (but associative) algebra holds.

For theories based on the Nambu brackets, the constraint algebra is of the form

$$
\left[\mathcal{C}_{\mathrm{F}}, \mathcal{C}_{\mathrm{F}^{\prime}}, \mathcal{C}_{\mathrm{F}^{\prime \prime}}\right]=f_{\mathrm{FF}^{\prime} \mathrm{F}^{\prime \prime}}{ }^{\prime \prime \prime} \mathcal{C}_{\mathrm{F}^{\prime \prime \prime}}
$$

The constant $f$ 's here are manifestly antisymmetric in the first three indices (and in fact, upon lowering the other index, totally antisymmetric). These generalize the standard Lie algebra's

\footnotetext{
${ }^{20}$ Here $N=8$ is the amount of supersymmetry and M2 denotes a spatially 2- $d$ M-Theory brane.
} 
structure constants. Indeed one reason for using Nambu brackets rather than associators themselves is that this generalization of Lie algebra ensues. The analogue of Jacobi identity for these brackets as regards which notions of (bi)beables close algebraically is the Filippov identity [63]

$$
[A, B,[C, D, E]]=[[A, B, C], D, E]+[C,[A, B, D], E]+[C, D,[A, B, E]]
$$

There is then a direct parallel of the Jacobi identity working (1.10), by which the Filippov identity and subsequent use of each of (7.1), (7.2) or the corresponding notions of bibeable establishes that each of these notions algebraically closes. (7.1) are then quantities which associate with pairs of constraints, whereas (7.2) are presently quantities pairs of which associate with constraints. Bibeables are then quantities that associate with whichever first-class constraint and whichever beable.

\subsection{Master constraint program's double bracket}

The master constraint program arose in one variant of loop quantum gravity [155]. In this approach one has just the usual $Q^{\mathrm{A}}$ and $P_{\mathrm{A}}$ and thus just the usual underlying 2-slot brackets notion. However, one trades one's constraints for a single master constraint. This involves packaging all the constraints (whether or not first-class [155]) into a single object known as the master constraint

$$
\mathbb{M}:=\frac{1}{2} \sum_{\mathrm{C}, \mathrm{C}^{\prime}} \mathcal{C}_{\mathrm{C}} K^{\mathrm{CC}^{\prime}} \mathcal{C}_{\mathrm{C}^{\prime}}
$$

Here $K^{\mathrm{CC}^{\prime}}$ is a positive operator on the space of square-summable sequences over the index set $\mathrm{C}$. Then the constraint surface for $\mathbb{M}$ coincides with that for $\mathcal{C}_{C}=0$. The master constraint then forms a trivial constraint algebra with itself.

Moreover, the usual notion of observables/beables is here replaced by the double 2-slot bracket condition

$$
\left\{\mathrm{B}_{\mathrm{B}},\left\{B_{\mathrm{B}^{\prime}}, \mathbb{M}\right\}\right\} \approx 0
$$

for master constraint observables/beables. By the general identity

$$
\{F,\{F, \mathbb{M}\}\}_{\mathbb{M}=0}=\sum_{C, C^{\prime}}\left\{F, \mathcal{C}_{\mathrm{C}}\right\}_{\mathbb{M}=0} K^{\mathrm{AC}}\left\{F, \mathcal{C}_{\mathrm{C}^{\prime}}\right\}_{\mathbb{M}=0}
$$

the single master equation (7.3) is equivalent to the infinity of equations $\left\{B_{\mathrm{B}}, \mathcal{C}_{\mathrm{C}}\right\}_{\mathbb{M} \approx 0} \approx 0$. Thus the master equation precisely picks out weak (Dirac) observables/beables.

This notion indeed also produces a closed algebraic structure: $\left\{B_{\mathrm{B}}, B_{\mathrm{B}^{\prime}}\right\}$ obeys $(7.3)$ if the $B_{\mathrm{B}}$ do. This is by use within the subsequent object built from three Poisson brackets

$$
\left\{\left\{B_{\mathrm{B}}, B_{\mathrm{B}^{\prime}}\right\},\left\{B_{\mathrm{B}^{\prime \prime}}, \mathbb{M}\right\}\right\}
$$

of firstly the standard Jacobi identity followed by two uses of (7.3).

The idea in this program is then to quantize $\mathbb{M}$ itself.

\section{Conclusion: a collection of frontiers}

Types of constraint, types of gauge theory, types of zero, types of bracket have been discussed, and underlie a large number of variants on the definition of observables or beables in a wide range of (models of) gravitational theories. This remains an open field. For instance, the following three programs have largely not yet been considered at the quantum level. 
1. The consequences of Pons et al. classical level work [130, 131].

2. Dittrich's power series $[57,58]$.

3. Concrete examples of Histories Theory approaches with nontrivial diffeomorphisms [103, $141]$.

4. On the other hand, Halliwell's approach $[77,79,80]$ has not yet been applied to perturbative or exact midisuperspace models; the more advanced form of this [79, 80] has not even been applied to any examples with additional linear gauge constraints.

5. Halliwell and Marolf's [115] approaches remain to be compared in detail with each other. Does one of these confer greater advantages, do the two approaches produce compatible results?

6. Footnote 15 considers various further areas ripe for review that are not covered in the present review or recent previous ones $[131,152]$. One would hope then that the population of such recent reviews would grow by a further one or two due to someone else's further treatment of asymptotic/boundary/holographic observables/beables in gravitational theories.

7. As outlined in this review, the beables aspect of supergravity is still in its infancy.

8. This review only touches on one of many possible applications of observables/beables to M-theory: following on from the use of Nambu brackets in the Bagger-Lambert-Gustavsson action.

Resolving the quantum problem of beables (and in a manner consistent with the rest of the problem of time facets of Appendix A) remains a major and overarching open problem.

\section{A Facets of the problem of time}

The problem of time $[4,93,106]$ has 9 facets stemming together from the mismatch in time concepts between GR and QM. See [10] for a more detailed account of these and how they are each underlied by an aspect of background independence.

Facet 1. Frozen formalism problem. The Schrödinger picture manifestation is that GR's quantum wave equation - the Wheeler-DeWitt equation $\widehat{\mathcal{H}} \Psi=0$, is a stationary, i.e. timeless wave equation, like $\widehat{H} \Psi=E \Psi$ as opposed to $\widehat{H} \Psi=i \hbar \partial \Psi / \partial t$ for some notion of time $t$. This question is determined by the purely quadratic nature of GR's Hamiltonian constraint $\mathcal{H}$, and in turn by the Leibnizian 'there is no time for the universe as a whole' temporal relationalism principle. Strategies for resolving this facet include the following.

O. Perhaps a fundamental hidden/internal time that can be discovered within classical GR by canonical reformulation.

A. Perhaps GR has emergent rather than fundamental time. E.g. in the semiclassical regime, slow, heavy modes provide an approximate time by which the other fast, light modes evolve.

B. Instead one could see how much of physics can be done timelessly, e.g. via addressing solely questions of being, rather than becoming. For example, records theory [2, 7, 9] concerns whether a single instant contains pattern/correlation information, and whether a semblance of dynamics or history arises from this.

C. Perhaps histories are primary instead: histories theory. At the quantum level, this involves consistent sets of histories, fine- and coarse-graining operations and decoherence functionals for comparing pairs of histories. Some versions [97] have furthermore a classical precursor involving the histories brackets as outlined in Section 7.2.

One can furthermore combine schemes A-C [3, 77, 79] since i) histories contain records, ii) histories decohereing (self-measuring) gives semiclassicality, iii) the elusive question of which degrees of freedom decohere which others is addressed via where info is actually stored, i.e. where the records are. Such a combination underlies Halliwell's approach to the problem of time [77, 79] of Sections 3.3, 6.1 and 6.2.

D. Consider QM in the Heisenberg picture instead (cf. Section 6.1), whether as a more lucid choice or as exclusively the only choice for quantum gravity [137]. 
Facet 2 - configurational relationalism - concerns dynamics with a physically irrelevant group of transformations $\mathfrak{g}$ acting on configuration space $\mathfrak{q}$. This gives rise to constraints that are linear in the momenta. Configurational relationalism is resolved for relational particle mechanics by Barbour's best matching: solving the Lagrangian form of the linear constraints for the $\mathfrak{g}$-auxiliary variables themselves. (This is an example of Section 2 formal strategy 1.) However this remains unresolved as Wheeler's well-known thin Sandwich problem [23, 32] occurs in the case of GR, for which the linear constraint is $\mathcal{M}_{i}, \mathfrak{g}$ is $\operatorname{Diff}(\Sigma)$ and the $\mathfrak{g}$-auxiliary variables form the shift vector $N^{i}$.

Facet 3 is the constraint closure problem, i.e. whether the brackets of the constraints $\mathcal{H}$ and $\mathcal{M}_{i}$ do not produce further conditions as per (1.4)-(1.6).

Facet 4 is the problem of beables/observables as exposited in the Introduction.

Facet $\mathbf{5}$ is spacetime relationalism. Here a physically-irrelevant group of motions acts on spacetime $\mathfrak{m}$; this is usually $\operatorname{Diff}(\mathfrak{m})$. This problem picks up additional nontrivialities as regards representations and the measures in path-integral and histories formulations at the quantum level.

Facet 6 is what happens at the quantum level to GR's independence of foliation of spacetime by spaces. This corresponds to the theoretical scheme being able to encode arbitrarily moving families of observers.

Facet 7 concerns how classical spacetime is to be reconstructed [13] from space and/or discrete notions.

Note that the Dirac algebroid for $\mathcal{H}$ and $\mathcal{M}_{i}$ resolves all three of 3),6), 7) at the classical level. However even the semiclassical counterpart of these resolutions remains unknown.

Facet 8 is a collection [12] of global complications with each of timefunctions (that could refer to globality in time itself or in how time is defined over space), or the other facets above. (E.g. best matching, foliations and spacetime reconstruction are in general only local constructs and with strategies for resolving facets (e.g. only locally defined hidden and emergent times.)

Facet 9 is the multiple choice problem: that classically canonically equivalent formulations are in general unitarily inequivalent at the quantum level [73].

\section{Acknowledgements}

E.A. thanks close people, Julian Barbour, Jeremy Butterfield, Harvey Brown, Sean Gryb, Jonathan Halliwell, Philipp Höhn, Chris Isham, Flavio Mercati, Brian Pitts, Josep Maria Pons, Oliver Pooley, Donald Salisbury, Dimitri Vey, Hans Westman, Michael Wright and the anonymous referees for discussions, Jeremy Butterfield, John Barrow, Marc Lachiéze-Rey, Malcolm MacCallum, Don Page, Reza Tavakol, Juan Valiente-Kroon and Paulo Vargas-Moniz for help with my career, and DAMTP Cambridge, Perimeter Institute Waterloo and the University of New Brunswick Fredericton for hospitality at various points during the making of this review. This work started within my grant from the Foundational Questions Institute (FQXi) Fund, a donor-advised fund of the Silicon Valley Community Foundation on the basis of proposal FQXi-RFP3-1101 to the FQXi, administered via Theiss Research and the CNRS and held at APC Université Paris Diderot.

\section{References}

[1] Anastopoulos C., Continuous-time histories: observables, probabilities, phase space structure and the classical limit, J. Math. Phys. 42 (2001), 3225-3259, quant-ph/0008052.

[2] Anderson E., Records theory, Internat. J. Modern Phys. D 18 (2009), 635-667, arXiv:0709.1892.

[3] Anderson E., Approaching the problem of time with a combined semiclassical-records-histories scheme, Classical Quantum Gravity 29 (2012), 235015, 37 pages, arXiv:1204.2868. 
[4] Anderson E., Problem of time in quantum gravity, Ann. Phys. 524 (2012), 757-786, arXiv:1206.2403.

[5] Anderson E., The problem of time in quantum gravity, in Classical and Quantum Gravity: Theory, Analysis and Applications, Editor V.R. Frignanni, Nova, New York, 2012, 213-256, arXiv:1009.2157.

[6] Anderson E., Relational quadrilateralland. I. The classical theory, Internat. J. Modern Phys. D 23 (2014), 1450014, 75 pages, arXiv:1202.4186.

[7] Anderson E., The problem of time and quantum cosmology in the relational particle mechanics arena, arXiv:1111.1472.

[8] Anderson E., Machian time is to be abstracted from what change?, arXiv:1209.1266.

[9] Anderson E., Kendall's shape statistics as a classical realization of Barbour-type timeless records theory approach to quantum gravity, arXiv:1307.1923.

[10] Anderson E., Background independence, arXiv:1310.1524.

[11] Anderson E., Problem of time in slightly inhomogeneous cosmology, arXiv:1403.7583.

[12] Anderson E., Global problems of time in quantum gravity, in preparation.

[13] Anderson E., Mercati F., Classical Machian resolution of the spacetime reconstruction problem, arXiv:1311.6541.

[14] Anderson I.M., Torre C.G., Classification of local generalized symmetries for the vacuum Einstein equations, Comm. Math. Phys. 176 (1996), 479-539, gr-qc/9404030.

[15] Anderson J.L., Principles of relativity physics, Academic Press, New York, 1967.

[16] Anderson J.L., Bergmann P.G., Constraints in covariant field theories, Phys. Rev. 83 (1951), 1018-1025.

[17] Arnold V.I., Mathematical methods of classical mechanics, Graduate Texts in Mathematics, Vol. 60, Springer-Verlag, New York - Heidelberg, 1978.

[18] Ashtekar A., Lectures on nonperturbative canonical gravity, Advanced Series in Astrophysics and Cosmology, Vol. 6, World Sci. Publ., River Edge, NJ, 1991.

[19] Ashtekar A., Tate R., Uggla C., Minisuperspaces: observables and quantization, Internat. J. Modern Phys. D 2 (1993), 15-50, gr-qc/9302027.

[20] Bagger J., Lambert N., Modeling multiple M2-branes, Phys. Rev. D 75 (2007), 045020, 7 pages, hepth/0611108.

[21] Bagger J., Lambert N., Comments on multiple M2-branes, J. High Energy Phys. 2008 (2008), no. 2, 105, 15 pages, arXiv:0712.3738.

[22] Bagger J., Lambert N., Three-algebras and $\mathcal{N}=6$ Chern-Simons gauge theories, Phys. Rev. D 79 (2009), 025002, 8 pages, arXiv:0807.0163.

[23] Baierlein R.F., Sharp D.H., Wheeler J.A., Three-dimensional geometry as carrier of information about time, Phys. Rev. 126 (1962), 1864-1866.

[24] Barbour J., The end of time, Oxford University Press, Oxford, 2000.

[25] Barbour J., Foster B.Z., Ó Murchadha N., Relativity without relativity, Classical Quantum Gravity 19 (2002), 3217-3248, gr-qc/0012089.

[26] Barbour J.B., The timelessness of quantum gravity. I. The evidence from the classical theory, Classical Quantum Gravity 11 (1994), 2853-2873.

[27] Barbour J.B., The timelessness of quantum gravity. II. The appearance of dynamics in static configurations, Classical Quantum Gravity 11 (1994), 2875-2897.

[28] Barbour J.B., Bertotti B., Mach's principle and the structure of dynamical theories, Proc. Roy. Soc. London Ser. A 382 (1982), 295-306.

[29] Barbour J.B., Foster B.Z., Constraints and gauge transformations: Dirac's theorem is not always valid, arXiv:0808.1223.

[30] Bardeen J.M., Gauge-invariant cosmological perturbations, Phys. Rev. D 22 (1980), 1882-1905.

[31] Barrett J., Wigner's friend and Bell's field beables, in Vision of Oneness, Editors I. Licata, A. Sakaji, Aracne, Rome, 2011, 63-82.

[32] Bartnik R., Fodor G., On the restricted validity of the thin sandwich conjecture, Phys. Rev. D 48 (1993), 3596-3599.

[33] Batalin I.A., Tyutin I.V., Existence theorem for the effective gauge algebra in the generalized canonical formalism with abelian conversion of second-class constraints, Internat. J. Modern Phys. A 6 (1991), 32553282 . 
[34] Bell J.S., Quantum mechanics for cosmologists, in Second Oxford Symposium on Quantum Gravity, Editors C.J. Isham, R. Penrose, D.W. Sciama, Clarendon, Oxford, 1981, 611-637.

[35] Bell J.S., Beables for quantum field theory, in Quantum Implications, Editors B.J. Hiley, D. Peat, Routledge \& Kegan Paul, London, 1987, 227-234.

[36] Bell J.S., The theory of local beables, in Speakable and Unspeakable in Quantum Mechanics, Cambridge University Press, Cambridge, 1987, 52-62.

[37] Belot G., Earman J., Pre-socratic quantum gravity, in Physics Meets Philosophy at the Planck Scale, Editors C. Callendar, N. Huggett, Cambridge University Press, Cambridge, 2001, 213-255.

[38] Bergmann P.G., "Gauge-invariant" variables in general relativity, Phys. Rev. 124 (1961), 274-278.

[39] Bergmann P.G., Komar A., The coordinate group symmetries of general relativity, Internat. J. Theoret. Phys. 5 (1972), 15-28.

[40] Berman D.S., Cederwall M., Kleinschmidt A., Thompson D.C., The gauge structure of generalised diffeomorphisms, J. High Energy Phys. 2013 (2013), no. 1, 064, 22 pages, arXiv:1208.5884.

[41] Bleecker D., Gauge theory and variational principles, Dover, New York, 2001.

[42] Bojowald M., Canonical gravity and applications: cosmology, black holes, and quantum gravity, Cambridge University Press, Cambridge, 2011.

[43] Bojowald M., Höhn P.A., Tsobanjan A., An effective approach to the problem of time, Classical Quantum Gravity 28 (2011), 035006, 18 pages, arXiv:1009.5953.

[44] Bojowald M., Höhn P.A., Tsobanjan A., Effective approach to the problem of time: general features and examples, Phys. Rev. D 83 (2011), 125023, 38 pages, arXiv:1011.3040.

[45] Bub J., Why not take all observables as beables?, in Fundamental Problems in Quantum Theory (Baltimore, MD, 1994), Ann. New York Acad. Sci., Vol. 755, New York Acad. Sci., New York, 1995, 761-767.

[46] Cannas da Silva A., Weinstein A., Geometric models for noncommutative algebras, Berkeley Mathematics Lecture Notes, Vol. 10, Amer. Math. Soc., Providence, RI, 1999.

[47] Carlip S., Observables, gauge invariance, and time in (2+1)-dimensional quantum gravity, Phys. Rev. D 42 (1990), 2647-2654.

[48] Carlip S., Measuring the metric in (2+1)-dimensional quantum gravity, Classical Quantum Gravity 8 (1991), $5-17$.

[49] Carlip S., Quantum gravity: a progress report, Rep. Progr. Phys. 64 (2001), 885-942, gr-qc/0108040.

[50] Casalbuoni R., On the quantization of systems with anticommuting variables, Nuovo Cimento A 33 (1976), $115-125$.

[51] Clifton R., Beables in algebraic quantum theory, in From Physics to Philosophy (Cambridge, 1997), Editors M. Redhead, J. Butterfield, C. Pagonis, Cambridge University Press, Cambridge, 1999, 12-43.

[52] D'Eath P.D., Supersymmetric quantum cosmology, Cambridge Monographs on Mathematical Physics, Cambridge University Press, Cambridge, 1996.

[53] Deser S., Kay J.H., Stelle K.S., Hamiltonian formulation of supergravity, Phys. Rev. D 16 (1977), 2448-2455.

[54] DeWitt B.S., The quantization of geometry, in Gravitation: An Introduction to Current Research, Editor L. Witten, Wiley, New York, 1962, 266-381.

[55] Dirac P.A.M., Forms of relativistic dynamics, Rev. Modern Phys. 21 (1949), 392-399.

[56] Dirac P.A.M., Lectures on quantum mechanics, Belfer Graduate School of Science Monographs Series, Vol. 2, Belfer Graduate School of Science, New York, 1964.

[57] Dittrich B., Partial and complete observables for canonical general relativity, Classical Quantum Gravity 23 (2006), 6155-6184, gr-qc/0507106.

[58] Dittrich B., Partial and complete observables for Hamiltonian constrained systems, Gen. Relativity Gravitation 39 (2007), 1891-1927, gr-qc/0411013.

[59] Dittrich B., Tambornino J., Gauge-invariant perturbations around symmetry-reduced sectors of general relativity: applications to cosmology, Classical Quantum Gravity 24 (2007), 4543-4585, gr-qc/0702093.

[60] Dittrich B., Tambornino J., A perturbative approach to Dirac observables and their spacetime algebra, Classical Quantum Gravity 24 (2007), 757-783, gr-qc/0610060.

[61] Döring A., Isham C., "What is a thing?": topos theory in the foundations of physics, in New Structures for Physics, Lecture Notes in Phys., Vol. 813, Editor R. Coecke, Springer, Heidelberg, 2011, 753-937, arXiv:0803.0417. 
[62] Earman J., Gauge matters, Philos. Sci. 69 (2002), S209-S220.

[63] Filippov V.T., n-Lie algebras, Sib. Math. J. 26 (1985), 879-891.

[64] Fradkin E.S., Vasiliev M.A., Hamiltonian formalism, quantization and $S$ matrix for supergravity, Phys. Lett. B 72 (1977), 70-74.

[65] Gambini R., Porto R.A., Relational time in generally covariant quantum systems: four models, Phys. Rev. D 63 (2001), 105014, 15 pages, gr-qc/0101057.

[66] Gambini R., Porto R.A., Pullin J., Torterolo S., Conditional probabilities with Dirac observables and the problem of time in quantum gravity, Phys. Rev. D 79 (2009), 041501, 5 pages, arXiv:0809.4235.

[67] Gambini R., Pullin J., Loops, knots, gauge theories and quantum gravity, Cambridge Monographs on Mathematical Physics, Cambridge University Press, Cambridge, 1996.

[68] Gambini R., Pullin J., Consistent discrete space-time, in 100 Years of Relativity. Space-Time Structure: Einstein and Beyond, Editor A. Ashtekar, World Sci. Publ., Hackensack, NJ, 2005, 415-444.

[69] Géhéniau J., Debever R., Les quatorze invariants de courbure de l'espace Riemannien a quatre dimensions, Helv. Phys. Acta Suppl. 4 (1956), 101-105.

[70] Gell-Mann M., Hartle J.B., Classical equations for quantum systems, Phys. Rev. D 47 (1993), 3345-3382, gr-qc/9210010.

[71] Giddings S.B., Marolf D., Hartle J.B., Observables in effective gravity, Phys. Rev. D 74 (2006), 064018, 20 pages, hep-th/0512200.

[72] Goldstein S., Norsen T., Tausk D.V., Zanghi N., Bell's theorem, Scholarpedia 6 (2011), 8378.

[73] Gotay M.J., Obstructions to quantization, in Mechanics: from Theory to Computation (Essays in Honor of Juan-Carlos Simó, Editors J. Marsden, S. Wiggins, Springer, New York, 2000, 171-216, math-ph/9809011.

[74] Gustavsson A., Algebraic structures on parallel M2 branes, Nuclear Phys. B 811 (2009), 66-76, arXiv:0709.1260.

[75] Hájíček P., Group quantization of parametrized systems. I. Time levels, J. Math. Phys. 36 (1995), 4612-4638, gr-qc/9412047.

[76] Hájíček P., Isham C.J., Perennials and the group-theoretical quantization of a parametrized scalar field on a curved background, J. Math. Phys. 37 (1996), 3522-3538, gr-qc/9510034.

[77] Halliwell J., The interpretation of quantum cosmology and the problem of time, in The Future of the Theoretical Physics and Cosmology (Cambridge, 2002), Editors G.W. Gibbons, E.P.S. Shellard, S.J. Rankin, Cambridge University Press, Cambridge, 2003, 675-692, gr-qc/0208018.

[78] Halliwell J.J., Somewhere in the universe: where is the information stored when histories decohere?, Phys. Rev. D 60 (1999), 105031, 17 pages, quant-ph/9902008.

[79] Halliwell J.J., Probabilities in quantum cosmological models: a decoherent histories analysis using a complex potential, Phys. Rev. D 80 (2009), 124032, 21 pages, arXiv:0909.2597.

[80] Halliwell J.J., Decoherent histories analysis of minisuperspace quantum cosmology, J. Phys. Conf. Ser. 306 (2011), 012023, 22 pages, arXiv:1108.5991.

[81] Halliwell J.J., Hawking S.W., Origin of structure in the Universe, Phys. Rev. D 31 (1985), 1777-1791.

[82] Halliwell J.J., Thorwart J., Life in an energy eigenstate: decoherent histories analysis of a model timeless universe, Phys. Rev. D 65 (2002), 104009, 19 pages, gr-qc/0201070.

[83] Halvorson H., Clifton R., Maximal beable subalgebras of quantum mechanical observables, Internat. J. Theoret. Phys. 38 (1999), 2441-2484, quant-ph/9905042.

[84] Hartle J.B., Spacetime quantum mechanics and the quantum mechanics of spacetime, in Gravitation et Quantifications (Les Houches, 1992), Editors B. Julia, J. Zinn-Justin, North-Holland, Amsterdam, 1995, 285-480, gr-qc/9304006.

[85] Hawking S.W., Laflamme R., Lyons G.W., Origin of time asymmetry, Phys. Rev. D 47 (1993), 5342-5356, gr-qc/9301017.

[86] Hawking S.W., Page D.N., Operator ordering and the flatness of the universe, Nuclear Phys. B 264 (1986), $185-196$.

[87] Hélein F., Kouneiher J., The notion of observable in the covariant Hamiltonian formalism for the calculus of variations with several variables, Adv. Theor. Math. Phys. 8 (2004), 735-777, math-ph/0401047.

[88] Henneaux M., Teitelboim C., Quantization of gauge systems, Princeton University Press, Princeton, NJ, 1992. 
[89] Höhn P.A., Effective relational dynamics, J. Phys. Conf. Ser. 360 (2012), 012014, 4 pages, arXiv:1110.5631.

[90] Höhn P.A., From classical to quantum: new canonical tools for the dynamics of gravity, Ph.D. Thesis, Utrecht University, 2012.

[91] Höhn P.A., Kubalová E., Tsobanjan A., Effective relational dynamics of a nonintegrable cosmological model, Phys. Rev. D 86 (2012), 065014, 21 pages, arXiv:1111.5193.

[92] Isham C.J., Topological and global aspects of quantum theory, in Relativity, Groups and Topology, II (Les Houches, 1983), Editors B. DeWitt, R. Stora, North-Holland, Amsterdam, 1984, 1059-1290.

[93] Isham C.J., Canonical quantum gravity and the problem of time, in Integrable Systems, Quantum Groups, and Quantum Field Theories (Salamanca, 1992), NATO Adv. Sci. Inst. Ser. C Math. Phys. Sci., Vol. 409, Editors L.A. Ibort, M.A. Rodríguez, Kluwer Acad. Publ., Dordrecht, 1993, 157-287, gr-qc/9210011.

[94] Isham C.J., Lectures on quantum theory. Mathematical and structural foundations, Imperial College Press, London, 1995.

[95] Isham C.J., Kuchar̆ K.V., Representations of spacetime diffeomorphisms. I. Canonical parametrized field theories, Ann. Physics 164 (1985), 288-315.

[96] Isham C.J., Kuchař K.V., Representations of spacetime diffeomorphisms. II. Canonical geometrodynamics, Ann. Physics 164 (1985), 316-333.

[97] Isham C.J., Linden N., Continuous histories and the history group in generalized quantum theory, J. Math. Phys. 36 (1995), 5392-5408, gr-qc/9503063.

[98] Jizba P., Pons J.M., Revisiting the gauge principle: enforcing constants of motion as constraints, J. Phys. A: Math. Theor. 43 (2010), 205202, 20 pages, arXiv:0905.3807.

[99] Joos E., Zeh H.D., The emergence of classical properties through interaction with the environment, Z. Phys. B 59 (1985), 223-243.

[100] Joos E., Zeh H.D., Kiefer C., Giulini D., Kupsch J., Stamatescu I.O., Decoherence and the appearance of a classical world in quantum theory, 2nd ed., Springer-Verlag, Berlin, 2003.

[101] Kasuya M., The Einstein-Cartan theory of gravitation in a Hamiltonian form, Progr. Theoret. Phys. 60 (1978), 167-177.

[102] Kent A., Might quantum-induced deviations from the Einstein equations detectably affect gravitational wave propagation?, Found. Phys. 43 (2013), 707-718, arXiv:1204.5961.

[103] Kouletsis I., Covariance and time regained in canonical general relativity, Phys. Rev. D 78 (2008), 064014, 22 pages, arXiv:0803.0125.

[104] Kuchař K.V., A bubble-time canonical formalism for geometrodynamics, J. Math. Phys. 13 (1972), 768-781.

[105] Kuchař K.V., General relativity: dynamics without symmetry, J. Math. Phys. 22 (1981), 2640-2654.

[106] Kuchař K.V., Time and interpretations of quantum gravity, in Proceedings of the 4th Canadian Conference on General Relativity and Relativistic Astrophysics (Winnipeg, MB, 1991), Editors G. Kunstatter, D. Vincent, J. Williams, World Sci. Publ., River Edge, NJ, 1992, 211-314.

[107] Kuchař K.V., Canonical quantum gravity, in General Relativity and Gravitation 1992 (Córdoba), Editors R.J. Gleiser, C.N. Kozamah, O.M. Moreschi, Institute of Physics Publishing, Bristol, 1993, 119-150, gr-qc/9304012.

[108] Kuchař K.V., Geometrodynamics of Schwarzschild black holes, Phys. Rev. D 50 (1994), 3961-3981, gr-qc/9403003.

[109] Kuchař K.V., The problem of time in quantum geometrodynamics, in The Arguments of Time, Editor J. Butterfield, Oxford University Press, Oxford, 1999, 169-196.

[110] Lanczos C., The variational principles of mechanics, University of Toronto Press, Toronto, Ont., 1949.

[111] Langlois D., Hamiltonian formalism and gauge invariance for linear perturbations in inflation, Classical Quantum Gravity 11 (1994), 389-407.

[112] Lee J., Wald R.M., Local symmetries and constraints, J. Math. Phys. 31 (1990), 725-743.

[113] Littlejohn R.G., Reinsch M., Gauge fields in the separation of rotations and internal motions in the $n$-body problem, Rev. Modern Phys. 69 (1997), 213-275.

[114] Lusanna L., Pauri M., The physical role of gravitational and gauge degrees of freedom in general relativity. II. Dirac versus Bergmann observables and the objectivity of space-time, Gen. Relativity Gravitation 38 (2006), 229-267, gr-qc/0407007.

[115] Marolf D., Solving the problem of time in mini-superspace: measurement of Dirac observables, Phys. Rev. D 79 (2009), 084016, 10 pages, arXiv:0902.1551. 
[116] Maudlin J., Quantum non-locality and relativity: metaphysical intimations of modern physics, Blackwell, Oxford, 2002.

[117] Mukhanov V.F., Feldman H.A., Brandenberger R.H., Theory of cosmological perturbations, Phys. Rep. 215 (1992), 203-333.

[118] Nambu Y., Generalized Hamiltonian dynamics, Phys. Rev. D 7 (1973), 2405-2412.

[119] Page D.N., Sensible quantum mechanics: are probabilities only in the mind?, Internat. J. Modern Phys. D 5 (1996), 583-596, gr-qc/9507024.

[120] Page D.N., Consciousness and the quantum, arXiv:1102.5339.

[121] Page D.N., Wootters W.K., Evolution without evolution: dynamics described by stationary observables, Phys. Rev. D 27 (1983), 2885-2892.

[122] Penrose R., Rindler W., Spinors and space-time. Vol. 1. Two-spinor calculus and relativistic fields, Cambridge Monographs on Mathematical Physics, Cambridge University Press, Cambridge, 1984.

[123] Penrose R., Rindler W., Spinors and space-time. Vol. 2. Spinor and twistor methods in space-time geometry, Cambridge Monographs on Mathematical Physics, Cambridge University Press, Cambridge, 1986.

[124] Pilati M., The canonical formulation of supergravity, Nuclear Phys. B 132 (1978), 138-154.

[125] Pitts J.B., A first class constraint generates not a gauge transformation, but a bad physical change: the case of electromagnetism, arXiv:1310.2756.

[126] Polyakov A.M., Gauge transformations and diffeomorphisms, Internat. J. Modern Phys. A 5 (1990), 833842.

[127] Pons J.M., Salisbury D.C., Issue of time in generally covariant theories and the Komar-Bergmann approach to observables in general relativity, Phys. Rev. D 71 (2005), 124012, 16 pages, gr-qc/0503013.

[128] Pons J.M., Salisbury D.C., Shepley L.C., Gauge transformations in the Lagrangian and Hamiltonian formalisms of generally covariant theories, Phys. Rev. D 55 (1997), 658-668, gr-qc/9612037.

[129] Pons J.M., Salisbury D.C., Sundermeyer K.A., Gravitational observables, intrinsic coordinates, and canonical maps, Modern Phys. Lett. A 24 (2009), 725-732, arXiv:0902.0401.

[130] Pons J.M., Salisbury D.C., Sundermeyer K.A., Revisiting observables in generally covariant theories in the light of gauge fixing methods, Phys. Rev. D 80 (2009), 084015, 23 pages, arXiv:0905.4564.

[131] Pons J.M., Salisbury D.C., Sundermeyer K.A., Observables in classical canonical gravity: folklore demystified, J. Phys. Conf. Ser. 222 (2010), 012018, 15 pages, arXiv:1001.2726.

[132] Rovelli C., Is there incompatibility between the ways time is treated in general relativity and in standard quantum mechanics?, in Conceptual Problems of Quantum Gravity (North Andover, MA, 1988), Einstein Stud., Vol. 2, Editors A. Ashtekar, J. Stachel, Birkhäuser Boston, Boston, MA, 1991, 126-140.

[133] Rovelli C., Quantum evolving constants. Reply to: "Comment on: 'Time in quantum gravity: an hypothesis'", Phys. Rev. D 44 (1991), 1339-1341.

[134] Rovelli C., Time in quantum gravity: an hypothesis, Phys. Rev. D 43 (1991), 442-456.

[135] Rovelli C., GPS observables in general relativity, Phys. Rev. D 65 (2002), 044017, 6 pages, gr-qc/0110003.

[136] Rovelli C., Partial observables, Phys. Rev. D 65 (2002), 124013, 8 pages, gr-qc/0110035.

[137] Rovelli C., Quantum gravity, Cambridge Monographs on Mathematical Physics, Cambridge University Press, Cambridge, 2004.

[138] Rovelli C., Why gauge?, Found. Phys. 44 (2014), 91-104, arXiv:1308.5599.

[139] Saunders S., The "beables" of relativistic pilot-wave theory, in From Physics to Philosophy (Cambridge, 1997), Editors M. Redhead, J. Butterfield, C. Pagonis, Cambridge University Press, Cambridge, 1999, 71-89.

[140] Savvidou N., Histories approach to general relativity. I. The spacetime character of the canonical description, Classical Quantum Gravity 21 (2004), 615-630, gr-qc/0306034.

[141] Savvidou N., Histories approach to general relativity. II. Invariance groups, Classical Quantum Gravity 21 (2004), 631-646, gr-qc/0306036.

[142] Schlosshauer M., Decoherence, the measurement problem, and interpretations of quantum mechanics, Rev. Modern Phys. 76 (2005), 1267-1305.

[143] Shirai I., Wada S., Cosmological perturbations and quantum fields in curved space, Nuclear Phys. B 303 (1988), 728-750.

[144] Smolin L., The present moment in quantum cosmology: challenges to the arguments for the elimination of time, in Time and the Instant, Editor R. Durie, Clinamen Press, Manchester, 2000, 112-143, gr-qc/0104097. 
[145] Smolin L., A real ensemble interpretation of quantum mechanics, Found. Phys. 42 (2012), 1239-1261, arXiv:1104.2822.

[146] Śniatycki J., Dirac brackets in geometric dynamics, Ann. Inst. H. Poincaré Sect. A 20 (1974), 365-372.

[147] Stewart J., Advanced general relativity, Cambridge Monographs on Mathematical Physics, Cambridge University Press, Cambridge, 1990.

[148] Struyve W., Pilot-wave theory and quantum fields, Rep. Progr. Phys. 73 (2010), 106001, 30 pages, arXiv:0707.3685.

[149] Szekeres P., The gravitational compass, J. Math. Phys. 6 (1965), 1387-1391.

[150] 't Hooft G., Determinism beneath quantum mechanics, in Quo Vadis Quantum Mechanics? (Philadelphia, 2002), Editors A. Elitzur, S. Dolev, N. Kolenda, Frontiers Collection, Springer-Verlag, Berlin, 2005, 99-111, quant-ph/0212095.

[151] Takhtajan L., On foundation of the generalized Nambu mechanics, Comm. Math. Phys. 160 (1994), 295315 , hep-th/9301111.

[152] Tambornino J., Relational observables in gravity: a review, SIGMA 8 (2012), 017,30 pages, arXiv:1109.0740.

[153] Teitelboim C., How commutators of constraints reflect the spacetime structure, Ann. Physics 79 (1973), $542-557$.

[154] Teitelboim C., Supergravity and square roots of constraints, Phys. Rev. Lett. 38 (1977), 1106-1110.

[155] Thiemann T., Modern canonical quantum general relativity, Cambridge Monographs on Mathematical Physics, Cambridge University Press, Cambridge, 2007.

[156] Torre C.G., Gravitational observables and local symmetries, Phys. Rev. D 48 (1993), R2373-R2376, gr-qc/9306030.

[157] Torre C.G., Observables for the polarized Gowdy model, Classical Quantum Gravity 23 (2006), 1543-1555, gr-qc/0508008.

[158] Torre C.G., Anderson I.M., Symmetries of the Einstein equations, Phys. Rev. Lett. 70 (1993), 3525-3529, gr-qc/9302033.

[159] Unruh W.G., Wald R.M., Time and the interpretation of canonical quantum gravity, Phys. Rev. D 40 (1989), 2598-2614.

[160] Vaisman I., Lectures on the geometry of Poisson manifolds, Progress in Mathematics, Vol. 118, Birkhäuser Verlag, Basel, 1994.

[161] Vargas Moniz P., Quantum Cosmology - the supersymmetric perspective. Vol. 1. Fundamentals, Lecture Notes in Phys., Vol. 803, Springer, Berlin, 2010.

[162] Vink J.C., Quantum mechanics in terms of discrete beables, Phys. Rev. A 48 (1993), 1808-1818.

[163] Wada S., Consistency of canonical quantization of gravity and boundary conditions for the wave function of the Universe, Phys. Rev. D 34 (1986), 2272-2276.

[164] Wada S., Quantum cosmological perturbations in pure gravity, Nuclear Phys. B 276 (1986), 729-743, Erratum, Nuclear Phys. B 284 (1987), 747-748.

[165] Wallace D., Philosophy of quantum mechanics, in The Ashgate Companion to Contemporary Philosophy of Physics, Editor D. Rickles, Ashgate, Aldershot, 2008, 16-98, arXiv:0712.0149.

[166] Weinberg S., The quantum theory of fields. Vol. I. Foundations, Cambridge University Press, Cambridge, 2005.

[167] Weinberg S., The quantum theory of fields. Vol. II. Modern applications, Cambridge University Press, Cambridge, 2005.

[168] Wheeler J.A., Superspace and the nature of quantum geometrodynamics, in Battelle Rencontres: 1967 Lectures in Mathematics and Physics, Editors C. DeWitt, J.A. Wheeler, Benjamin, New York, 1968, 242307.

[169] Wüthrich C., Approaching the Planck scale from a generally relativistic point of view: a philosophical appraisal of loop quantum gravity, Ph.D. Thesis, University of Pittsburgh, 2006. 